PAPER • OPEN ACCESS

\title{
Strain relaxation dynamics of multiferroic orthorhombic manganites
}

To cite this article: M A Carpenter et al 2021 J. Phys.: Condens. Matter 33125402

View the article online for updates and enhancements.

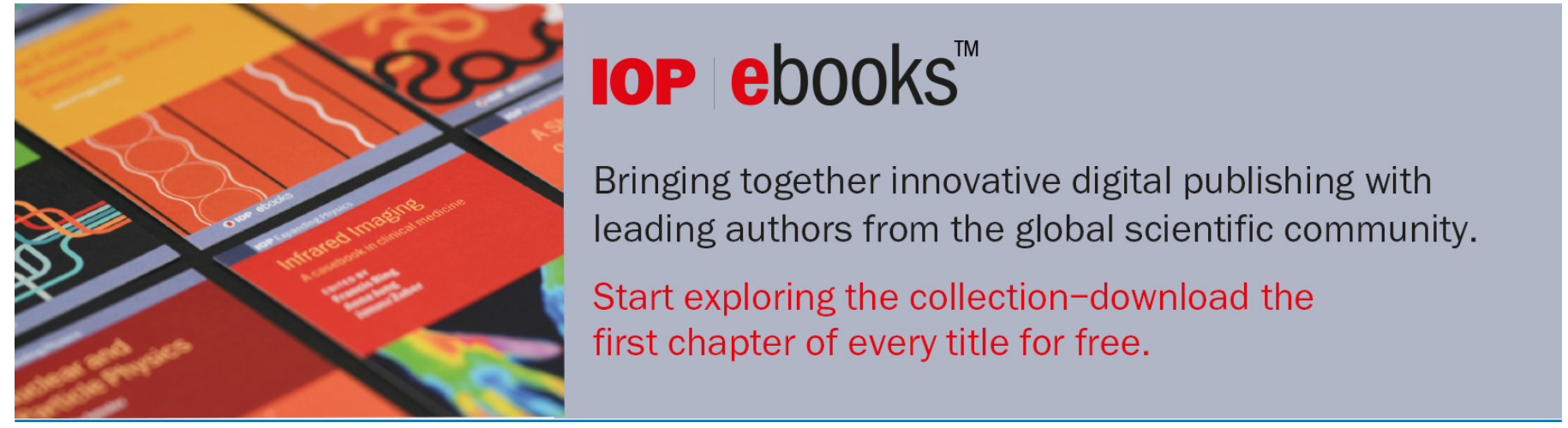

This content was downloaded from IP address 137.205 .164 .220 on 12/04/2021 at 15:30 


\title{
Strain relaxation dynamics of multiferroic orthorhombic manganites
}

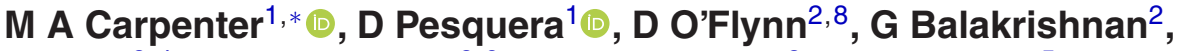

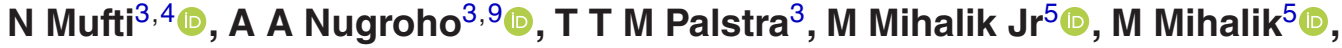 \\ M Zentková ${ }^{5}$, A Almeida ${ }^{6}$, J Agostinho Moreira ${ }^{6}{ }^{\infty}$, R Vilarinho $^{6}$ and D \\ Meier $^{7}$ (1) \\ ${ }^{1}$ Department of Earth Sciences, University of Cambridge, Downing Street, Cambridge CB2 3EQ, United \\ Kingdom \\ ${ }^{2}$ Department of Physics, University of Warwick, Coventry CV4 7AL, United Kingdom \\ 3 Solid State Chemistry Laboratory, Zernike Institute for Advanced Materials, Rijksuniversiteit \\ Groningen, Nijenborgh 4, 9747AG Groningen, The Netherlands \\ ${ }^{4}$ Department of Physics, Universitas Negeri Malang, Jl. Semarang No.5, Malang, 65145 Indonesia, \\ Indonesia \\ 5 Institute of Experimental Physics, Slovak Academy of Sciences, Watsonova 47, Košice, Slovakia \\ ${ }^{6}$ IFIMUP, Departamento de Física e Astronomia, Faculdade de Ciências da Universidade do Porto, Rua \\ do Campo Alegre 687, 4169-007 Porto, Portugal \\ 7 Department of Materials Science and Engineering, NTNU Norwegian University of Science and \\ Technology, 7491 Trondheim, Norway \\ E-mail:mc43@esc.cam.ac.uk
}

Received 4 August 2020, revised 14 September 2020

Accepted for publication 2 October 2020

Published 8 April 2021

\begin{abstract}
Resonant ultrasound spectroscopy has been used to characterise strain coupling and relaxation behavior associated with magnetic/magnetoelectric phase transitions in $\mathrm{GdMnO}_{3}, \mathrm{TbMnO}_{3}$ and $\mathrm{TbMn}_{0.98} \mathrm{Fe}_{0.02} \mathrm{O}_{3}$ through their influence on elastic/anelastic properties. Acoustic attenuation ahead of the paramagnetic to colinear-sinusoidal incommensurate antiferromagnetic transition at $\sim 41 \mathrm{~K}$ correlates with anomalies in dielectric properties and is interpreted in terms of Debye-like freezing processes. A loss peak at $\sim 150 \mathrm{~K}$ is related to a steep increase in electrical conductivity with a polaron mechanism. The activation energy, $E_{\mathrm{a}}$, of $\gtrsim 0.04 \mathrm{eV}$ from a loss peak at $\sim 80 \mathrm{~K}$ is consistent with the existence of a well-defined temperature interval in which the paramagnetic structure is stabilised by local, dynamic correlations of electric and magnetic polarisation that couple with strain and have relaxation times in the vicinity of $\sim 10^{-6} \mathrm{~s}$. Comparison with previously published data for $\mathrm{Sm}_{0.6} \mathrm{Y}_{0.4} \mathrm{MnO}_{3}$ confirms that this pattern may be typical for multiferroic orthorhombic $R \mathrm{MnO}_{3}$ perovskites $(R=\mathrm{Gd}, \mathrm{Tb}, \mathrm{Dy})$. A frequency-dependent loss peak near $10 \mathrm{~K}$ observed for $\mathrm{TbMnO}_{3}$ and $\mathrm{TbMn}_{0.98} \mathrm{Fe}_{0.02} \mathrm{O}_{3}$, but not for $\mathrm{GdMnO}_{3}$, yielded $E_{\mathrm{a}} \geqslant$ $\sim 0.002 \mathrm{eV}$ and is interpreted as freezing of some magnetoelastic component of the cycloid structure. Small anomalies in elastic properties associated with the incommensurate and cycloidal magnetic transitions confirm results from thermal expansion data that the magnetic

\footnotetext{
* Author to whom any correspondence should be addressed.

${ }^{8}$ Current address: Department of Scientific Research, The British Museum, Great Russell Street, London WC1B 3DG, United Kingdom

${ }^{9}$ Current Address: Faculty of Mathematics and Natural Sciences, Institut Teknologi Bandung Jl. Ganesha 10, Bandung, 40132 Indonesia. Indonesia
}

(c) (i) Original content from this work may be used under the terms of the Creative Commons Attribution 4.0 licence. Any further distribution of this work must maintain attribution to the author(s) and the title of the work, journal citation and DOI. 
order parameters have weak but significant coupling with strain. Even at strain magnitudes of $\sim 0.1-1 \%$, polaron-like strain effects are clearly important in defining the development and evolution of magnetoelectric properties in these materials. Strains associated with the cubic-orthorhombic transition due to the combined Jahn-Teller/octahedral tilting transition in the vicinity of $1500 \mathrm{~K}$ are $2-3$ orders of magnitude greater. It is inevitable that ferroelastic twin walls due to this transition would have significantly different magnetoelectric properties from homogeneous domains due to magnetoelastic coupling with steep strain gradients.

Keywords: multiferroics, strain coupling, magnetoelastic relaxation

(Some figures may appear in colour only in the online journal)

\section{Introduction}

Rare earth orthomanganites $\left(R \mathrm{MnO}_{3}, R=\right.$ rare earth) are well known for the multiple phase transitions which they display, including cooperative Jahn-Teller distortions, charge ordering, octahedral tilting, magnetism, ferroelectricity and magnetoelectricity. However, only $\mathrm{GdMnO}_{3}, \mathrm{TbMnO}_{3}$ and $\mathrm{DyMnO}_{3}$ have $\mathrm{Mn}-\mathrm{O}-\mathrm{Mn}$ bond angles which fall in the narrow range that is required for multiferroic properties where ferroelectricity arises from cycloidal magnetism [1-4]. It is clear that sensitivity to the size of the A-site cation and the lattice distortions which follow are a vital component of the structural and magnetic stability relationships even though strain is not the functional property of primary interest. On the other hand, the role of strain is exploited in the preparation of thin films where choice of substrate material provides a tuning mechanism for the magnetoelectric properties of all three materials (e.g. [5-11]). Strain/order parameter coupling has a fundamental influence on phase transitions, of course, in that it promotes mean field behavior by enhancing the interaction length of the order parameter and provides an indirect mechanism by which multiple order parameters can couple. Strain coupling is important also at ferroelastic domain walls where steep gradients in the primary order parameter(s) interact with equivalently steep gradients in strain, with the result that the walls can have properties which are substantially different from those of the immediately adjacent homogeneous domains. Consideration of domain walls as providing engineered functional properties in their own right has become a new focus for development of nanoscale devices more generally (e.g. [12-16]). Finally, the dynamic response of both thin films and bulk materials to some externally applied magnetic or electric field may be constrained by the dynamics of strain relaxation as the rate limiting step. For example, switching of domains from positive to negative polarity typically requires motion of the domain walls, which may depend on the mobility of polaronic-type strain clouds or unpinning of walls from the strain fields of point defects.

Against this wider view of strain coupling, the primary objective of the present study was to investigate the dynamics of strain relaxation associated specifically with the multiferroic behavior of $\mathrm{GdMnO}_{3}$ and $\mathrm{TbMnO}_{3}$. There are numerous studies of the temperature and frequency dependence of the dielectric responses of both materials to ac electric fields (including $[2,3,17-28]$ ) and one of the dynamical response to an ac magnetic field [29], but there appear to be no data in the literature for the elastic/anelastic response to ac stress. Significant differences have been observed between acoustic resonance spectra of hexagonal $\mathrm{YMnO}_{3}$ and orthorhombic $\mathrm{Sm}_{0.6} \mathrm{Y}_{0.4} \mathrm{MnO}_{3}$, however, and these provided evidence of a strain relaxation process that is specific to the multiferroic orthorhombic structure $[30,31]$. Both hexagonal $\mathrm{YMnO}_{3}$ and orthorhombic $\mathrm{Sm}_{0.6} \mathrm{Y}_{0.4} \mathrm{MnO}_{3}$ have spontaneous strains with magnitudes of up to $\sim 0.0004$ associated with antiferromagnetic ordering below $\sim 70 \mathrm{~K}$ and $\sim 50 \mathrm{~K}$, respectively, and an increase in attenuation above $\sim 200 \mathrm{~K}$ which is most likely related to increasing electrical conductivity. However, $\mathrm{Sm}_{0.6} \mathrm{Y}_{0.4} \mathrm{MnO}_{3}$ has two additional Debye-like peaks in acoustic attenuation at $\sim 80$ and $\sim 150 \mathrm{~K}$, preceding the incommensurate and cycloidal magnetic transitions [30].

There is no single technique which provides the mechanical equivalent of dielectric spectroscopy across decades of frequency, but resonant ultrasound spectroscopy (RUS) has proved to be effective for measuring the elastic and anelastic properties of mm-sized samples in the frequency range $\sim 0.1-2 \mathrm{MHz}$ [32]. Variations of resonance frequencies and peak widths provide, in particular, a measure of the strength and dynamics of strain coupling in perovskites which undergo conventional ferroic phase transitions [33].

Here we show that acoustic loss peaks obtained from analysis of RUS spectra from single crystals of $\mathrm{GdMnO}_{3}, \mathrm{TbMnO}_{3}$ and $\mathrm{TbMn}_{0.98} \mathrm{Fe}_{0.02} \mathrm{O}_{3}$ reveal a temperature interval between $\sim 40$ and $\sim 200 \mathrm{~K}$ in which dynamical strain relaxation occurs on a time scale of $\sim 10^{-6} \mathrm{~s}$. A loss peak at $\sim 80 \mathrm{~K}$ complements evidence of local electric dipole motion from dielectric spectroscopy and matches up with anomalies in thermal conductivity and thermal expansion. There appears to be a dynamical precursor structure which has coupling between local electric dipoles, strain and magnetic ordering. Kinetic parameters obtained from fitting of the inverse mechanical quality factor can be accounted for in terms of thermally activated relaxation of polaron-type strain clouds. The static magnetic structures become stable only once these become immobile. There is a further magnetoelastic loss process associated with ordering of moments of $\mathrm{Tb}^{3+}$ in the cycloid structure but not of $\mathrm{Gd}^{3+}$ in the A-type antiferromagnetic structure. Elastic stiffening below $\sim 41 \mathrm{~K}$ due to coupling of the magnetic order parameters with strain, rather than softening, confirms that their relaxation in response to applied stress occurs on a significantly longer time scale than $\sim 10^{-6} \mathrm{~s}$ in the stability field 


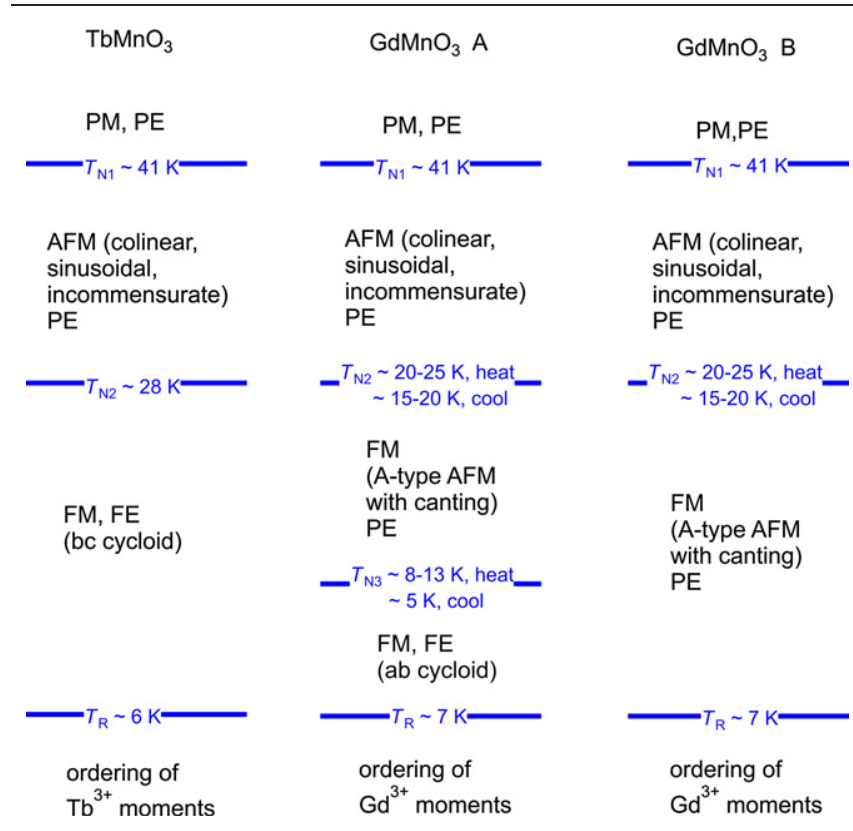

Figure 1. Summary from the literature of transition sequences with falling temperatures in $\mathrm{TbMnO}_{3}$ and $\mathrm{GdMnO}_{3}$, shown here as reference for comparison with complex patterns of elastic and anelastic behavior from RUS measurements. $\mathrm{PM}=$ paramagnetic, $\mathrm{PE}=$ paraelectric, $\mathrm{AFM}=$ antiferromagnetic, $\mathrm{WFM}=$ weakly ferromagnetic, $\mathrm{FE}=$ ferroelectric. $\mathrm{A}$ and $\mathrm{B}$ describe two different transition sequences observed in $\mathrm{GdMnO}_{3}$.

of the multiferroic phase. Differences in the sequence of magnetic structures between $\mathrm{GdMnO}_{3}$ and $\mathrm{TbMnO}_{3}$ are also seen clearly in the evolution of their elastic properties.

\section{Transition sequences}

$\mathrm{GdMnO}_{3}$ and $\mathrm{TbMnO}_{3}$ are orthorhombic in space group Pbnm at room temperature. Symmetry reduction from the holosymmetric cubic perovskite structure is due to combined octahedral tilting and cooperative Jahn-Teller distortions. The transition temperature for the cooperative Jahn-Teller transition is in the vicinity of $1500 \mathrm{~K}$ [34].

For comparison with the elastic and anelastic properties reported here, the sequences of magnetic transitions which occur in these materials at low temperatures are summarised in figure 1. Both undergo a second order, paramagnetic to colinear-sinusoidal incommensurate antiferromagnetic phase transition at $T_{\mathrm{N} 1} \approx 41 \mathrm{~K}$, with [010] as the direction of the temperature-dependent incommensurate repeat [1, 35-39]. The second transition is referred to here as occurring at $T_{\mathrm{N} 2}$. It is thermodynamically continuous at $\sim 28 \mathrm{~K}$ in $\mathrm{TbMnO}_{3}$, and leads to a structure with a magnetic cycloid in the (100) plane (' $b c$-cycloid'). The repeat parallel to [010] locks in to a nearly constant wavelength, and a ferroelectric polarisation develops parallel to [001] [1-4, 17, 36, 37]. More recently, Mufti et al [40] showed a small discontinuity in the development of the electric polarisation at $\sim 27 \mathrm{~K}$, suggesting weakly first order character for the transition. The second transition in $\mathrm{GdMnO}_{3}$ is to the A-type antiferromagnetic structure, with canting of Mn moments resulting in a net ferromagnetic moment parallel to [001], and is first order in character $[1-4,17,39$,
$41,42]$. The measured value of $T_{\mathrm{N} 2}$ differs between heating, $\sim 20-25 \mathrm{~K}$, and cooling, $15-20 \mathrm{~K}[2,3,17,43]$.

Some samples of $\mathrm{GdMnO}_{3}$ have an additional first order transition, referred to here as occurring at $T_{\mathrm{N} 3}\left(\mathrm{GdMnO}_{3} \mathrm{~A}\right.$ in figure 1). In zero magnetic field, $T_{\mathrm{N} 3}$ might be $\sim 10-13 \mathrm{~K}$ on heating and $\sim 5 \mathrm{~K}$ on cooling $[17,44]$ or $\sim 8 \mathrm{~K}$ on heating and $\sim 5 \mathrm{~K}$ on cooling $[2,3]$. The transition is associated with the development of electric polarisation parallel to [100] $[3,4,17,45]$ of a commensurate magnetic cycloid in the $(001)$ plane ('ab-cycloid') $[45,46]$. The $a b$-cycloid structure is stabilised by applying a magnetic field parallel to [010] [3, 39]. In the phase diagrams of Arima et al [39] and Baier et al [47] it is shown as being not quite stable in zero field. Goto et al [4] suggested that differences between samples in this regard may be due to slight differences in oxygen stoichiometry.

Ordering of the $\mathrm{Gd}$ and $\mathrm{Tb}$ moments occurs below a temperature $T_{\mathrm{R}}$ which is $\sim 7$ and $\sim 6 \mathrm{~K}$, respectively, in $\mathrm{GdMnO}_{3}$ and $\mathrm{TbMnO}_{3}$ [1, 2, 35-38, 47-49]. GdMnO 3 can retain the ferroelectric polarisation below $T_{\mathrm{R}}$ [45], but the phase diagrams of Goto et al [4] and Kimura et al [3] show a stability field for the weakly ferromagnetic, paraelectric structure instead. The phase diagram of Baier et al [47] has a field of phase coexistence. Hemberger et al [41] showed the low temperature structure as being antiferromagnetic with canting of $\mathrm{Gd}$ and $\mathrm{Mn}$ moments in opposite directions. In contrast, $\mathrm{TbMnO}_{3}$ retains the $b c$-cycloid structure down to the lowest temperatures. $T_{\mathrm{R}}$ is marked by the repeat distance parallel to [010] becoming fixed to a rational value [50] and by a slight increase in the electric polarisation parallel to $[001][2,3,36]$.

\section{Sample description}

The $\mathrm{GdMnO}_{3}$ single crystal used in the present study came from a boule grown by the floating zone method in air in the laboratory of M Mihalik. It had an irregular shape with maximum dimensions $\sim 3 \times 1 \times 1 \mathrm{~mm}^{3}$ and mass $0.0091 \mathrm{~g}$.

The first crystal of $\mathrm{TbMnO}_{3}$ ('crystal 1') was from a boule which had been grown in air by the floating zone technique [40]. Another crystal from the same boule was used in the study of Handayani et al [51]. The crystal used for RUS had mass $0.0084 \mathrm{~g}$, two parallel faces $\sim 0.75 \mathrm{~mm}$ apart and a triangular cross section with dimensions $\sim 1 \times \sim 2 \mathrm{~mm}^{2}$. Anomalies in the temperature dependence of electric polarisation, dielectric spectroscopy and lattice parameter data of Mufti et al [40] gave $T_{\mathrm{N} 2} \approx 26 \mathrm{~K}$. The value of $T_{\mathrm{N} 1}$ was quoted as $42 \mathrm{~K}$ and there was a break in slope of the temperature dependence of electric polarisation at $\sim 8 \mathrm{~K}$.

In order to test for the effects of small variations in oxygen stoichiometry, a second $\mathrm{TbMnO}_{3}$ crystal ('crystal 2') was examined. This came from the same boule as the crystal described by O'Flynn et al [52], which had been grown by the floating zone technique in argon atmosphere at the University of Warwick, and used for studies reported in that paper. On the basis of measurements of dc magnetic susceptibility and heat capacity it had transition temperatures $T_{\mathrm{N} 1}=41 \mathrm{~K}$, $T_{\mathrm{N} 2}=27 \mathrm{~K}, T_{\mathrm{R}} \approx 7 \mathrm{~K}$. The shape was approximately that of a rectangular parallelepiped, with dimensions $\sim 2.9 \times 1.9 \times 0.9 \mathrm{~mm}^{3}$, and mass $0.0296 \mathrm{~g}$. 
Substitution of $\mathrm{Mn}^{3+}$ by $\mathrm{Fe}^{3+}$ has been of interest because it allows, in principle, tuning of the contribution of Jahn-Teller cooperative distortions [53-56]. The two cations have closely similar radii but $\mathrm{Fe}^{3+}$ is not Jahn-Teller active. The single crystal of $\mathrm{TbMn}_{0.98} \mathrm{Fe}_{0.02} \mathrm{O}_{3}$ used in the present study was a slice with mass $0.1184 \mathrm{~g}$ cut from the same boule as described by Mihalik et al [54], which had been grown by the floating zone method in air. It had two parallel faces $\sim 1.2 \mathrm{~mm}$ apart and an irregular shape in the other two directions, with maximum dimensions of $\sim 3$ and $\sim 6 \mathrm{~mm}$. The transition temperatures reported by Mihalik et al [54]) on the basis of peaks in the heat capacity were $T_{\mathrm{N} 1}=38.4(3) \mathrm{K}, T_{\mathrm{N} 2}=21.8(3) \mathrm{K}$, $T_{\mathrm{R}} \approx 6.5 \mathrm{~K}$.

Elasticity and anelasticity data from these crystals are compared below with previous results obtained by RUS from a single crystal of $\mathrm{Sm}_{0.6} \mathrm{Y}_{0.4} \mathrm{MnO}_{3}$ [30] which has a sequence of structures closely similar to that of $\mathrm{TbMnO}_{3}[57,58]$. The sinusoidal antiferromagnetic structure developed below $T_{\mathrm{N} 1} \approx 50 \mathrm{~K}$, followed by $b c$-cycloidal ordering and the continuous development of a ferroelectric dipole parallel to [001] below $T_{\mathrm{N} 2} \approx 27 \mathrm{~K}$. A broad anomaly in heat capacity at the lowest temperatures was assumed to be due to ordering of Sm moments below $T_{\mathrm{R}} \approx 4 \mathrm{~K}$.

\section{Experimental methods}

The RUS technique has been described in detail by Migliori and Sarrao [32]. In the Cambridge set up, RUS data are collected with a crystal held lightly between two piezoelectric transducers in a head which is attached to a stick lowered into a helium flow cryostat [59] or an Oxford instruments cryogenfree Teslatron [60]. Maximum voltages applied to the driving transducer are 25 and $2 \mathrm{~V}$, respectively. For the present study, spectra were collected in automated cooling and heating sequences with a few mbars of helium gas in the sample chamber to assist thermal equilibration. A settle time of 20 min was allowed at each set point before data collection. Primary spectra contained between 65000 and 13000 points in different frequency intervals between 0.1 and $2 \mathrm{MHz}$.

Separate peaks in an RUS spectrum are due to natural acoustic resonances of the sample and involve predominantly shearing motions. For a single resonance peak with frequency $f$, values of the corresponding combination of (shear) elastic moduli scale with $f^{2}$. Assignment of specific combinations of moduli to specific resonances is generally possible only if the shape of the sample is well defined, such as for a rectangular parallelepiped. Line broadening arises as a consequence of acoustic loss, which is expressed in terms of the inverse mechanical quality factor, $Q^{-1}$. In an RUS experiment it is convenient to take $Q^{-1}$ as being equal to $\Delta f / f$, where $\Delta f$ is the peak width at half maximum height. Absolute values of $f^{2}$ and $Q^{-1}$ for resonances of a single crystal will display different temperature dependences through a phase transition, depending primarily on the manner in which different shear strains are coupled with the order parameter and with defects responsible for the loss processes. For the present study, resonance peaks which could be followed through wide intervals of temperature were fit with an asymmetric Lorentzian function

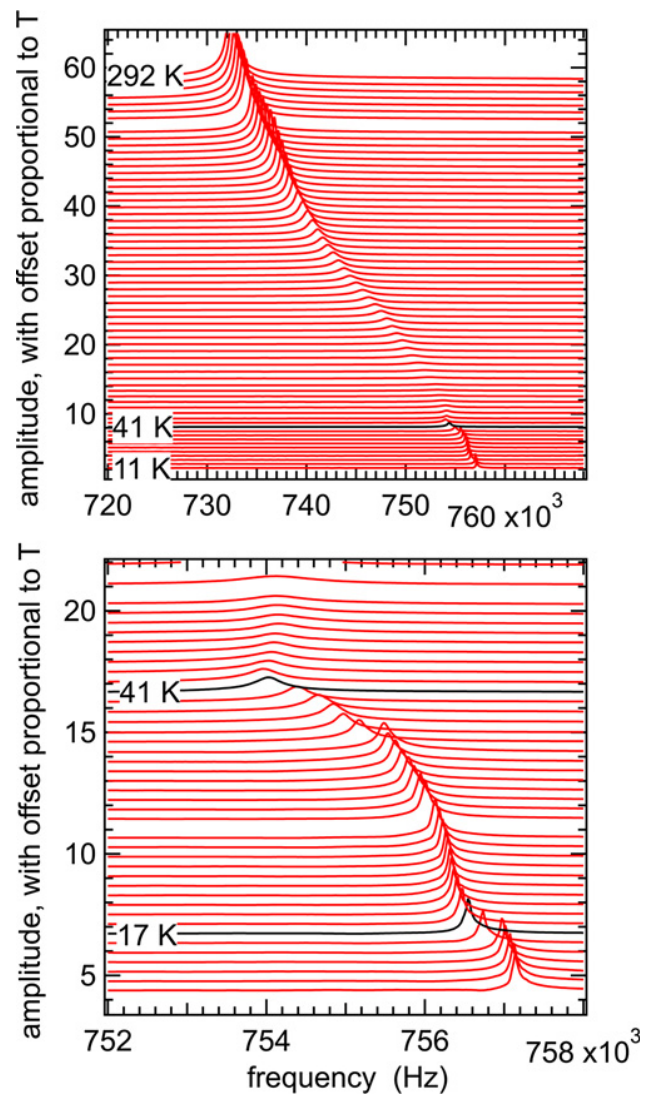

Figure 2. Segments of the RUS spectra from the single crystal of $\mathrm{GdMnO}_{3}$ collected during heating from 11 to $292 \mathrm{~K}$ in the helium flow cryostat. The spectra are offset up the $y$-axis in proportion to the temperature at which they were collected. They show the temperature dependence of a single resonance peak with frequency near $730 \mathrm{kHz}$ at room temperature.

in the software package Igor (Wavemetrics) to obtain the peak frequency, $f$, and width at half maximum height, $\Delta f$.

An example of how resonances evolve with temperature is given in figure 2 for a heating sequence using the $\mathrm{GdMnO}_{3}$ single crystal. The pattern shown by the single peak is of increasing resonance frequency with decreasing temperature and small but distinct breaks in trend at $\sim 41$ and $\sim 17 \mathrm{~K}$, corresponding to the expected transition temperatures, $T_{\mathrm{N} 1}$ and $T_{\mathrm{N} 2}$. Marked peak broadening, corresponding to a peak in $Q^{-1}$ values, is evident between $\sim 200$ and $\sim 50 \mathrm{~K}$.

\section{Results}

\section{1. $\mathrm{GdMnO}_{3}$ crystal}

Figure 3 shows results for $f^{2}$ and $Q^{-1}$ from fitting of resonance peaks in spectra from the single crystal of $\mathrm{GdMnO}_{3}$ collected in a heating sequence using the helium flow cryostat. The automated sequence involved cooling in $30 \mathrm{~K}$ steps from $280 \mathrm{~K}$ down to $10 \mathrm{~K}$ followed by heating from 10 to $50 \mathrm{~K}$ in $1 \mathrm{~K}$ steps, from 50 to $70 \mathrm{~K}$ in $2 \mathrm{~K}$ steps and from 70 to $295 \mathrm{~K}$ in $5 \mathrm{~K}$ steps. Data for $f^{2}$ from seven resonance peaks have been scaled along the $y$-axis in an arbitrary manner in figure 3(a) so as to allow easy comparison of their temperature dependences. The clear overall trend is of increasing steepness of elastic stiffening with falling temperature, 


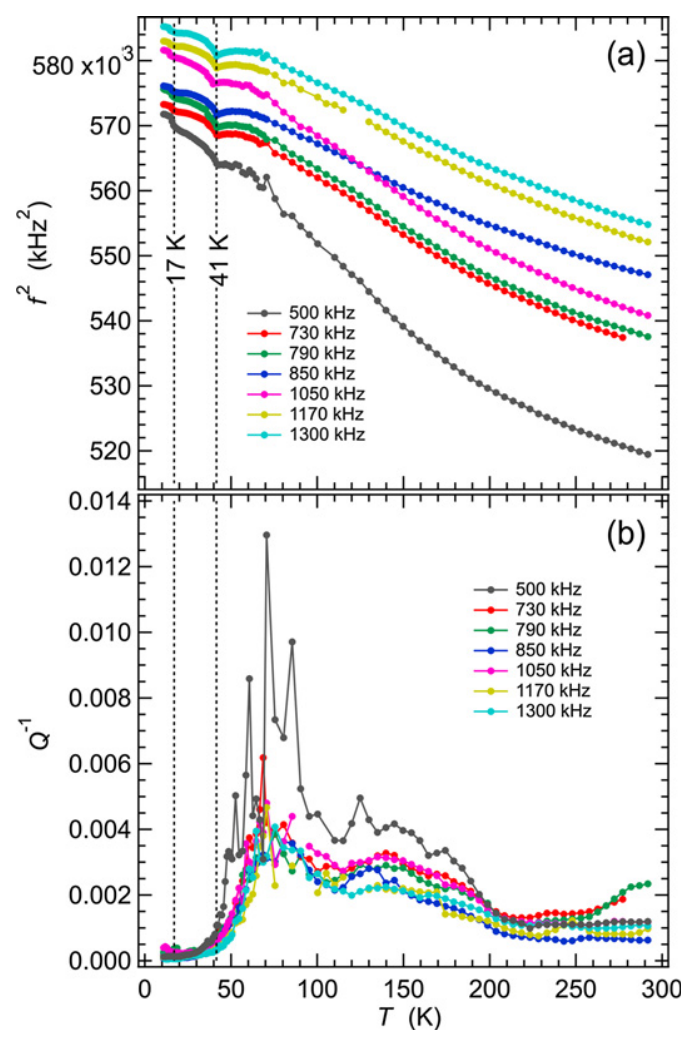

Figure 3. Variation of $f^{2}$ (a) and $Q^{-1}$ (b) for the single crystal of $\mathrm{GdMnO}_{3}$ from fitting of seven resonance peaks in RUS spectra collected during heating in the helium flow cryostat. Absolute values of $f^{2}$ have been scaled arbitrarily along the $y$-axis to allow for easy comparison of the trends which they each display. Listed frequencies refer to the approximate frequency of each resonance at room temperature. Scatter of the values of $Q^{-1}$ for the resonance peak with frequency near $500 \mathrm{kHz}$ is due to noise in the original spectra.

without the leveling off toward zero slope as $T \rightarrow 0 \mathrm{~K}$ expected for a normal crystalline material. Anomalies seen in the primary spectra (lower half of figure 2) are a small dip in $f^{2}$ at $T_{\mathrm{N} 1}$ and a small increase at $T_{\mathrm{N} 2}$. The different combinations of elastic moduli represented by $f^{2}$ for the different resonances vary by up to $\sim 10 \%$, with a dip of $\sim 0.4 \%$ at $T_{\mathrm{N} 1}$ and a small increase at $T_{\mathrm{N} 2}$.

The steepening trend of $f^{2}$ with falling temperature is accompanied by two broad peaks in $Q^{-1}$, centred at $\sim 80$ and $\sim 150 \mathrm{~K}$ (figure $3(\mathrm{~b})$ ), as is typical of Debye-like freezing processes. Resonance modes with the largest values of $Q^{-1}(f \sim 500$ and $1050 \mathrm{kHz}$ at room temperature $)$ also have the largest changes in $f^{2}$, consistent with this interpretation. $Q^{-1}$ drops to low values as $T_{\mathrm{N} 1}$ is approached from above and there is no obvious anomaly at $T_{\mathrm{N} 2}$. This pattern of acoustic loss is essentially the same for all resonances, from which it is concluded that all the single crystal moduli are affected in more or less the same way. This, in turn, implies that the loss mechanisms do not have a strong dependence on the orientation of the shear strain that applies in each resonance mode.

Data collected separately in the Teslatron cryostat extended to lower temperatures, as illustrated in figure 4 for a sequence of cooling followed by heating in small steps through the magnetic transitions. At this level of detail, the transition at $T_{\mathrm{N} 1}$ is fully reversible and occurs close to where $Q^{-1}$ drops to

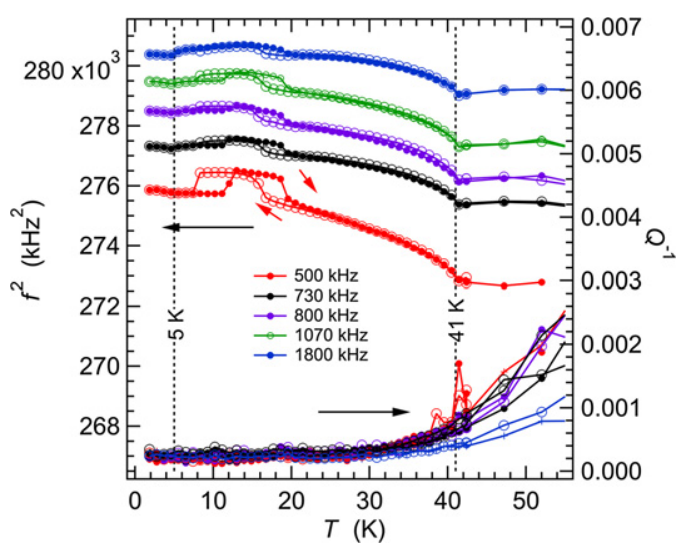

Figure 4. Variation of $f^{2}$ (a) and $Q^{-1}$ (b) at low temperatures from fitting of five resonance peaks in RUS spectra collected from the single crystal of $\mathrm{GdMnO}_{3}$ during cooling (open circles), followed by heating (filled circles) in the Teslatron cryostat. Absolute values of $f^{2}$ have been scaled arbitrarily along the $y$-axis to allow for easy comparison of the trends which they each display. Listed frequencies refer to the approximate frequency of each resonance at room temperature.

its lowest values. There is then a hysteretic transition in the expected region of $T_{\mathrm{N} 2}$, with a break in slope of $f^{2}$ occurring at $\sim 16 \mathrm{~K}$ during cooling and $\sim 19 \mathrm{~K}$ during heating. This is followed by a second additional hysteretic transition at $\sim 8 \mathrm{~K}$ during cooling and $\sim 12 \mathrm{~K}$ during heating, below which the resonance frequencies all revert back to the trend established above $T_{\mathrm{N} 2}$. The second hysteretic transition correlates with $T_{\mathrm{N} 3}$ in the pattern shown for $\mathrm{GdMnO}_{3} \mathrm{~A}$ in figure 1. On this basis, the A-type AFM structure stable between $T_{\mathrm{N} 2}$ and $T_{\mathrm{N} 3}$ is slightly stiffer than both the colinear-sinusoidal incommensurate structure (above $T_{\mathrm{N} 2}$ ) and the $a b$-cycloid structure (below $\left.T_{\mathrm{N} 3}\right)$.

Finally, there is a small reversible break in slope of the temperature dependences of $f^{2}$ at $\sim 5 \mathrm{~K}$. On the basis of studies in the literature, as summarised in figure 1, this appears to correspond to $T_{\mathrm{R}}$. Goto et al [4] and Kimura et al [3] showed a stability field for the weakly ferromagnetic, paraelectric structure below $T_{\mathrm{R}}$, i.e. that there is a transition from the $a b$-cycloid structure back to the canted antiferromagnetic structure with falling temperature. This would be consistent, in particular, with the data shown in figure 14 of Kimura et al [3] which show ferroelectric polarisation parallel to [100] only between $\sim 8$ and $\sim 5 \mathrm{~K}$. However, the RUS data in figure 4 do not show a return to the stiffer trend of the A-type AFM structure below $T_{\mathrm{R}}$, so the small anomaly may be due only to the development of long-range ordering of the magnetic moments of $\mathrm{Gd}^{3+}$.

\section{2. $\mathrm{TbMnO}_{3}$ crystal 1}

Figure 5 shows results for $f^{2}$ and $Q^{-1}$ from fitting of resonance peaks in spectra from $\mathrm{TbMnO}_{3}$, crystal 1, collected in a heating sequence using the helium flow cryostat. The full sequence involved cooling in $30 \mathrm{~K}$ steps at nominal temperatures from $280 \mathrm{~K}$ down to $10 \mathrm{~K}$ followed by heating from 10 to $60 \mathrm{~K}$ in $2 \mathrm{~K}$ steps and from 60 to $295 \mathrm{~K}$ in $5 \mathrm{~K}$ steps. In a second run, spectra were collected during heating from 120 to $210 \mathrm{~K}$ in $2 \mathrm{~K}$ steps. The data in figure 5(a) for $f^{2}$ from 8 resonance peaks 


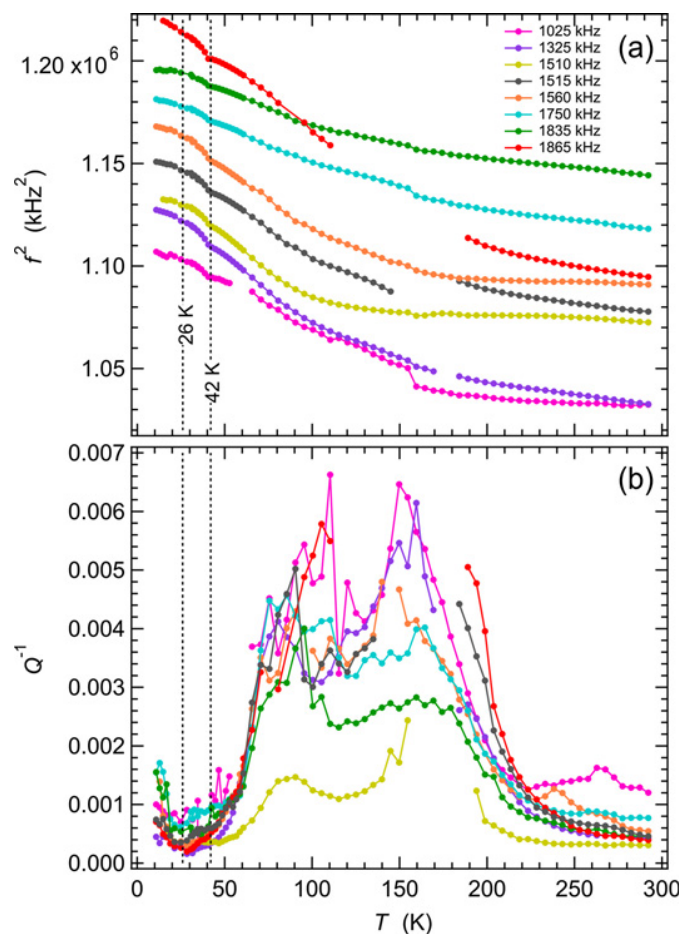

Figure 5. Variation of $f^{2}$ (a) and $Q^{-1}$ (b) during heating, from fitting of eight resonance peaks in RUS spectra collected in the helium flow cryostat from the first single crystal of $\mathrm{TbMnO}_{3}$ (crystal 1).

Absolute values of $f^{2}$ have been scaled arbitrarily along the $y$-axis to allow for easy comparison of the trends which they each display. Listed frequencies refer to the approximate frequency of each resonance at room temperature. The values of $T_{\mathrm{N} 1}$ and $T_{\mathrm{N} 2}$ shown are from Mufti et al [40]. Gaps and irregularities in the temperature dependence of both $f^{2}$ and $Q^{-1}$, particularly near $160 \mathrm{~K}$, occur where peaks in the primary spectra became too weak and broad to allow them to be fit with any confidence.

have again been scaled along the $y$-axis in an arbitrary manner to allow easy comparison of their temperature dependences.

As in the case of $\mathrm{GdMnO}_{3}$, the evolution of $f^{2}$ for each peak follows a steepening trend with falling temperature. However, the two resonances with frequencies near 1510 and $1560 \mathrm{kHz}$ at room temperature have a flat variation between 300 and $\sim 150 \mathrm{~K}$, which almost becomes a slight softening. The most obvious anomaly in the evolution of $f^{2}$ is a small dip at $41 \mathrm{~K}$, consistent with the value of $T_{\mathrm{N} 1} \approx 42$ given by Mufti et al [40]. There is perhaps a further small change in the temperature dependence of $f^{2}$ at the expected value of $T_{\mathrm{N} 2}=26 \mathrm{~K}$, but this is only at the level of noise. Overall, the elastic moduli vary by up to $\sim 10 \%$, and by a very small fraction of this at the transition temperatures. Small breaks in slope of $f^{2}$ near $160 \mathrm{~K}$ may or may not be real as they occurred in the interval where the peaks were broadest and hardest to follow.

There are again two broad peaks in $Q^{-1}$, centred at $\sim 80$ and $\sim 150 \mathrm{~K}$ (figure $5(\mathrm{~b})$ ), consistent with Debye-like freezing processes. The resonance modes with the largest values of $Q^{-1}$, i.e. those with frequencies near 1025,1325 and $1865 \mathrm{kHz}$ at room temperature, also have the largest changes in $f^{2}$, while the resonance with frequency near $1510 \mathrm{kHz}$ shows the lowest values of $Q^{-1}$ and the smallest overall change in $f^{2}$. These differences imply that there is some slight dependence of the loss mechanism on the orientation of induced strains but no

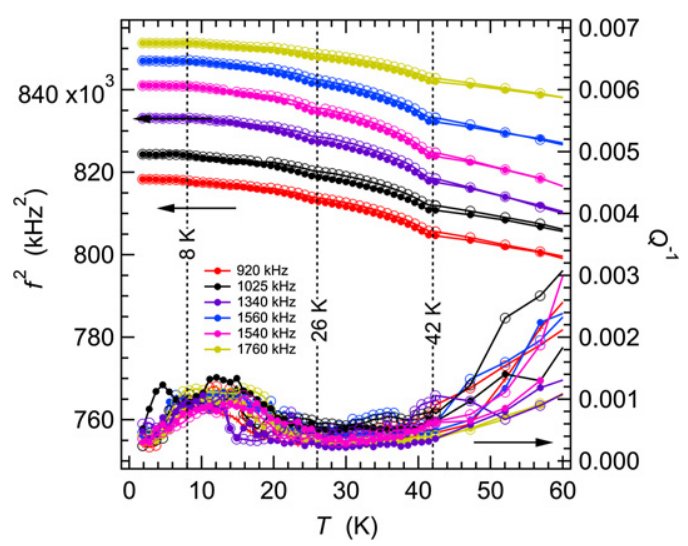

Figure 6. Variation of $f^{2}$ and $Q^{-1}$ at low temperatures from fitting of six resonance peaks in RUS spectra collected from $\mathrm{TbMnO}_{3}$ crystal 1 during cooling (open circles), followed by heating (filled circles) in the Teslatron cryostat. Absolute values of $f^{2}$ have been scaled arbitrarily along the $y$-axis to allow for easy comparison of the trends which they each display. Listed frequencies refer to the approximate frequency of each resonance at room temperature. The values of $T_{\mathrm{N} 1}$ and $T_{\mathrm{N} 2}$ shown are from Mufti et al [40], who also reported a break in slope of electric polarisation at $8 \mathrm{~K}$. Values of $T_{\mathrm{R}}$ from the literature are $\sim 6 \mathrm{~K}$ (figure 1, section 2 ).

obviously systematic dependence on frequency. $Q^{-1}$ drops to low values as $T_{\mathrm{N} 1}$ is approached from above and does not display any obvious anomaly at $T_{\mathrm{N} 2}$.

Data collected separately in the Teslatron cryostat extended to lower temperatures, as illustrated in figure 6 for a sequence of cooling followed by heating in small steps through the magnetic transitions. At this level of detail, the transition at $T_{\mathrm{N} 1}$ is reversible and again occurs essentially where $Q^{-1}$ drops to its lowest values. There is a very slight dip discernible in the evolution of $f^{2}$ at $\sim 26 \mathrm{~K}$, corresponding to the expected position of $T_{\mathrm{N} 2}$. As in the case of $\mathrm{GdMnO}_{3}$, there are no overt anomalies in $Q^{-1}$ associated with either of these two transitions. In contrast with $\mathrm{GdMnO}_{3}$, however, $Q^{-1}$ values extracted from all resonance peaks show a broad peak centred at $\sim 13 \mathrm{~K}$. This is above the value of $T_{\mathrm{R}} \approx 6 \mathrm{~K}$ reported in the literature and the temperature of $\sim 8 \mathrm{~K}$ where Mufti et al [40] observed a break in slope of the electric polarisation parallel to [001].

\section{3. $\mathrm{TbMnO}_{3}$ crystal 2}

Figure 7 contains $f^{2}$ and $Q^{-1}$ data for a representative set of resonance peaks collected from the second $\mathrm{TbMnO}_{3}$ crystal (crystal 2) in the Teslatron cryostat. Spectra were collected in a sequence of cooling followed by heating through the temperature interval 2-295 K. The pattern of variations of $f^{2}$ is closely similar to that of crystal 1 (figure 5), and is also fully reversible between cooling and heating. The pattern of variations of $Q^{-1}$ is different, however. Firstly, the peak in $Q^{-1}$ at $\sim 80 \mathrm{~K}$ of crystal 1 is still present but is not as obvious for all resonances from crystal 2 . Secondly, the peak at $\sim 150 \mathrm{~K}$ from crystal 1 is either absent in the data from crystal 2 or is hidden by the steep rise of $Q^{-1}$. Finally, $Q^{-1}$ returned to low values above $\sim 200 \mathrm{~K}$ for crystal 1 but continued to increase for crystal 2. There is perhaps a peak centred at $\sim 260 \mathrm{~K}$ in figure 7 (b). In addition, the maximum value of $Q^{-1}$ near $90 \mathrm{~K}$ varies more substantially between resonances, implying that the loss 


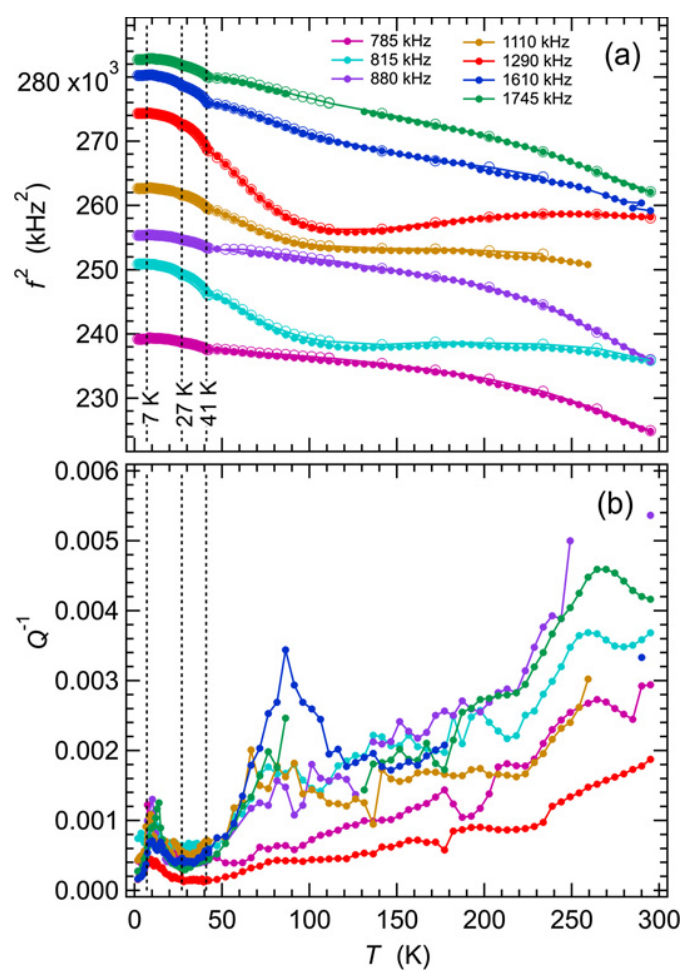

Figure 7. Variation of $f^{2}$ (a) and $Q^{-1}$ (b) from fitting of seven resonance peaks in RUS spectra collected during cooling (open circles) followed by heating (filled circles) in the Teslatron cryostat from the second single crystal of $\mathrm{TbMnO}_{3}$ (crystal 2). Absolute values of $f^{2}$ have been scaled arbitrarily along the $y$-axis to allow for easy comparison of the trends which they each display. Open circles in (a) indicate the cooling sequence and filled circles represent the heating sequence. Only data from the heating sequence are shown in (b). The data are indistinguishable between cooling and heating. Listed frequencies refer to the approximate frequency of each resonance at room temperature. Values shown for $T_{\mathrm{N} 1}, T_{\mathrm{N} 2}$ and $T_{\mathrm{R}}$ are from O'Flynn et al [52].

process is more sensitive to the orientation of the strain induced in each resonance mode. The resonance with frequency near $1290 \mathrm{kHz}$ actually softens with falling temperature down to $\sim 100 \mathrm{~K}$ and has the lowest values of $Q^{-1}$. All the resonance peaks displayed essentially the same peak in $Q^{-1}$ centred at $\sim 10 \mathrm{~K}$, a few degrees above the expected value of $T_{\mathrm{R}}$.

At the level of detail shown in figure 8 for the same data up to $60 \mathrm{~K}$, the transition at $T_{\mathrm{N} 1}$ is reversible and, as before, occurs essentially where $Q^{-1}$ drops to its lowest values. There is a very slight dip discernible in the evolution of $f^{2}$ at $\sim 27 \mathrm{~K}$, corresponding to the expected position of $T_{\mathrm{N} 2}$. There are, again, no overt anomalies in $Q^{-1}$ associated with either of these transitions. The peak in $Q^{-1}$ values at $\sim 10 \mathrm{~K}$ is observed for all resonances and there is a small softening step in $f^{2}$ values with falling temperature below $\sim 8 \mathrm{~K}$.

\section{4. $\mathrm{TbMn}_{0.98} \mathrm{Fe}_{0.02} \mathrm{O}_{3}$ crystal}

RUS spectra collected from the $\mathrm{TbMn}_{0.98} \mathrm{Fe}_{0.02} \mathrm{O}_{3}$ single crystal in an automated sequence involved cooling in $30 \mathrm{~K}$ steps at nominal temperatures from $280 \mathrm{~K}$ down to $10 \mathrm{~K}$ followed by heating from 10 to $70 \mathrm{~K}$ in $2 \mathrm{~K}$ steps and from 70 to 295 $\mathrm{K}$ in $5 \mathrm{~K}$ steps. Figure 9 shows results for $f^{2}$ and $Q^{-1}$ from

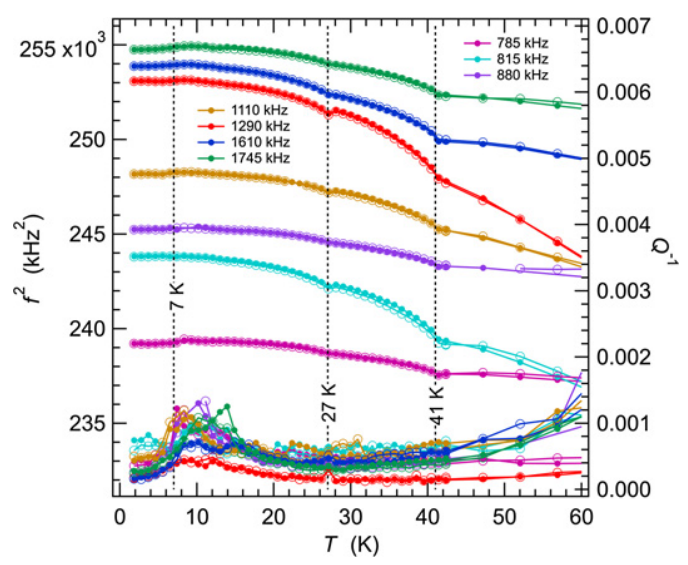

Figure 8. Enhanced view of variations of $f^{2}$ (a) and $Q^{-1}$ (b) data from figure 6 up to $60 \mathrm{~K}$. Absolute values of $f^{2}$ have been scaled arbitrarily along the $y$-axis to allow for easy comparison of the trends which they each display.

fitting of eleven resonance peaks. The overall pattern for some modes is of significant elastic softening with decreasing temperature followed by stiffening. The others show more uniform stiffening. The total variation is by up to $\sim 7 \%$, with a small dip at $T_{\mathrm{N} 1} \approx 38 \mathrm{~K}$ and an even smaller change in slope at $T_{\mathrm{N} 2} \approx 22 \mathrm{~K}$. There is a broad asymmetric peak in $Q^{-1}$ at $\sim 150 \mathrm{~K}$. Differences in the maximum values between different resonances imply that the loss process involves coupling with some specific orientation of induced strain. Subsequent measurements in the Teslatron cryostat showed that $f^{2}$ variations were fully reversible between cooling and heating.

Data collected separately in the Teslatron cryostat extended to lower temperatures, as illustrated in figure 10 for a sequence of cooling followed by heating in small steps through the magnetic transitions. As for all the other samples, the transition at $T_{\mathrm{N} 1}$ is reversible. There is a very slight, rounded dip discernible in the evolution of $f^{2}$ for the lowest frequency peak ( $\sim 270 \mathrm{kHz}$ ) at $\sim 22 \mathrm{~K}$, corresponding to the expected position of $T_{\mathrm{N} 2}$. In contrast with the other samples, there is a frequency dependent increase in $Q^{-1}$ starting at $\sim 30 \mathrm{~K}$ for the $1130 \mathrm{kHz}$ peak and at $\sim 22 \mathrm{~K}$ for the $270 \mathrm{kHz}$ peak. There is also an asymmetric peak in $Q^{-1}$ values with a maximum at $\sim 7-10 \mathrm{~K}$, which correlates with smooth but slight increases in $f^{2}$. There is no obvious anomaly in the evolution of $f^{2}$ at the expected value of $T_{\mathrm{R}} \sim 6.5 \mathrm{~K}$.

\section{Analysis}

\subsection{Debye loss peaks}

A thermally activated Debye freezing process observed in measurements made as a function of temperature at constant frequency can be described by (following [33, 61, 62])

$$
Q^{-1}(T)=Q_{\mathrm{m}}^{-1}\left[\cosh \left\{\frac{E_{\mathrm{a}}}{R r_{2}(\beta)}\left(\frac{1}{T}-\frac{1}{T_{\mathrm{m}}}\right)\right\}\right]^{-1} .
$$

The temperature, $T_{\mathrm{m}}$, at which $Q^{-1}$ has its maximum value, $Q_{\mathrm{m}}$, is determined by the condition $\omega \tau=1$, where $\tau$ is the relaxation time for the loss mechanism and $\omega$ is the angular 


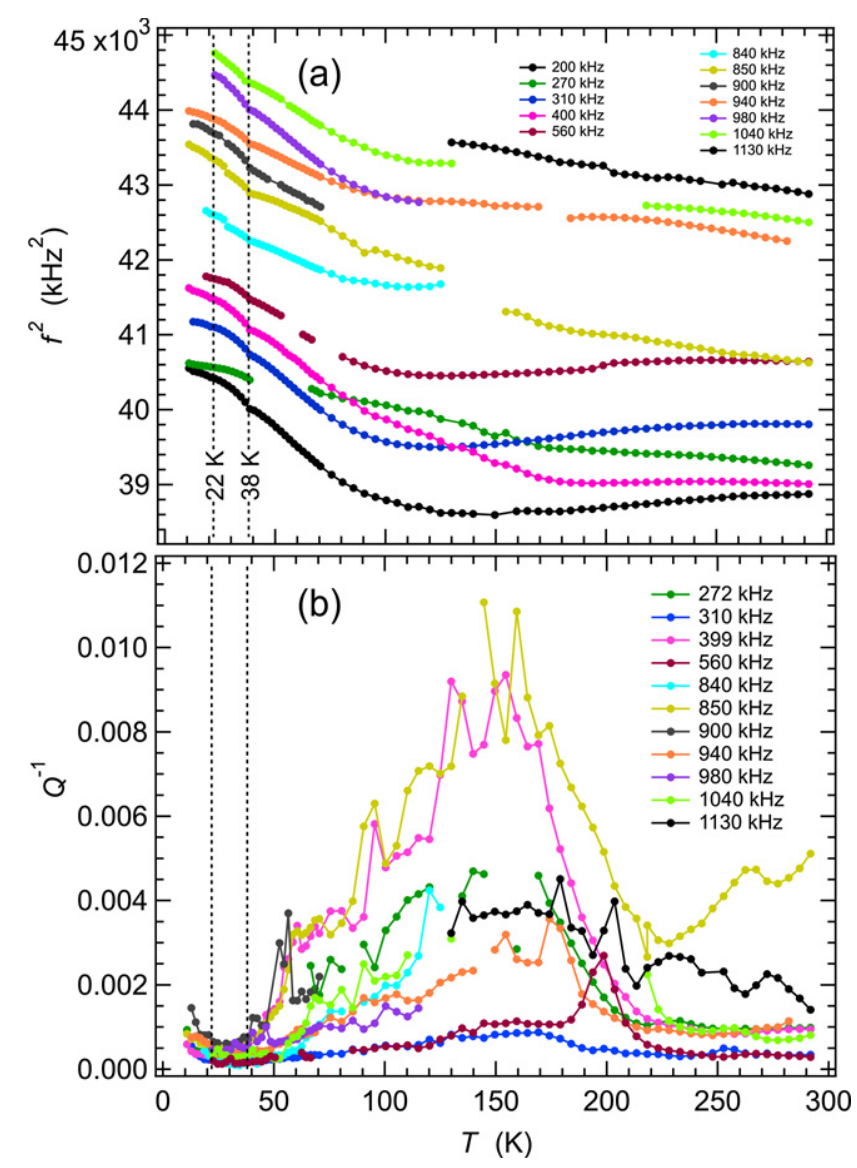

Figure 9. Variation of $f^{2}$ (a) and $Q^{-1}$ (b) during heating, from fitting of eleven resonance peaks in RUS spectra collected in the helium flow cryostat from the single crystal of $\mathrm{TbMn}_{0.98} \mathrm{Fe}_{0.02} \mathrm{O}_{3}$. Absolute values of $f^{2}$ have been scaled arbitrarily along the $y$-axis to allow for easy comparison of the trends which they each display. Listed frequencies refer to the approximate frequency of each resonance at room temperature. Values shown for $T_{\mathrm{N} 1}$ and $T_{\mathrm{N} 2}$ are from Mihalik et al [54]. Gaps and irregularities in the temperature dependence of both $f^{2}$ and $Q^{-1}$, particularly near $160 \mathrm{~K}$, occur where peaks in the primary spectra became too weak and broad to allow them to be fit with any confidence.

frequency $(=2 \pi f)$ at which the measurement is made. $E_{\mathrm{a}}$ is an activation energy, $R$ is the gas constant and $r_{2}(\beta)$ is a width parameter which defines a spread of relaxation times for the dissipation process.

Raw data for $Q^{-1}$ from four of the samples described above show evidence of overlapping peaks which, in lowest order, can be represented in terms of a single loss process with $T_{\mathrm{m}}$ in the vicinity of $10 \mathrm{~K}$ and two loss processes with $T_{\mathrm{m}}$ in the vicinities of 80 and $150 \mathrm{~K}$, respectively. Fits of equation (1) to the higher temperature data for selected resonances from the four samples investigated in the present study and from the single crystal of $\mathrm{Sm}_{0.6} \mathrm{Y}_{0.4} \mathrm{MnO}_{3}$ described elsewhere [30] are shown in figures 11(a)-(e). Reasonable fits to the separate peaks were generally obtained, though it was necessary to constrain some of the parameters due to overlaps and noise. The overall result is that a plausible description of the variations of $Q^{-1}$ for all five samples in the higher temperature range is in terms of two peaks with $E_{\mathrm{a}} / r_{2}(\beta) \approx 0.03-0.05$ and $0.08-0.1 \mathrm{eV}$. By somewhat arbitrarily combining data for

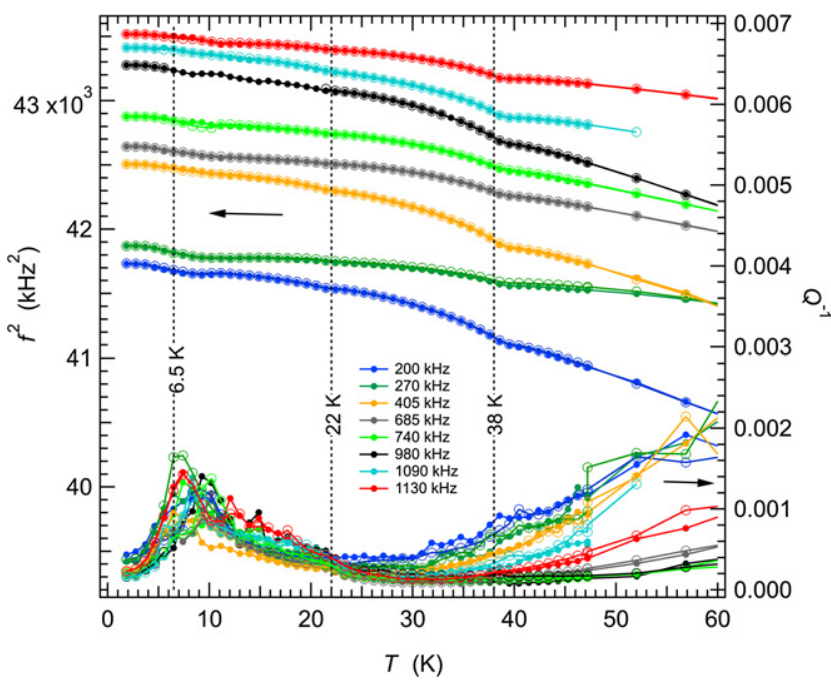

Figure 10. Variation of $f^{2}$ (a) and $Q^{-1}$ (b) at low temperatures from fitting of eight resonance peaks in RUS spectra collected from

$\mathrm{TbMn}_{0.98} \mathrm{Fe}_{0.02} \mathrm{O}_{3}$ during cooling (open circles), followed by heating (filled circles) in the Teslatron cryostat. Absolute values of $f^{2}$ have been scaled arbitrarily along the $y$-axis to allow for easy comparison of the trends which they each display. Listed frequencies refer to the approximate frequency of each resonance at room temperature. Values shown for $T_{\mathrm{N} 1}, T_{\mathrm{N} 2}$ and $T_{\mathrm{R}}$ are from Mihalik et al [54].

the different samples in a single Arrhenius plot (figure 11(f)), values of $T_{\mathrm{m}}$ for the loss peak near $80 \mathrm{~K}$ can be represented by $\tau=\tau_{\mathrm{o}} \exp \left(E_{\mathrm{a}} / \mathrm{R} T\right)$, with $\tau_{\mathrm{o}}=2.5 \times 10^{-10} \mathrm{~s}$, $E_{\mathrm{a}}=0.044 \pm 0.007 \mathrm{eV}$. In combination, the two fits would constrain $r_{2}(\beta)$ to be $\sim 1$ for the $80 \mathrm{~K}$ loss peak, implying that the relaxation mechanism involves a single relaxation time. Values of $T_{\mathrm{m}}$ from fits of equation (1) in the vicinity of $150 \mathrm{~K}$ do not produce the same quality of correlation and are therefore not shown. This is not surprizing given that the data indicate more than one loss process in different samples.

The steep increase in $Q^{-1}$ with increasing temperature above $\sim 250 \mathrm{~K}$ is present only in the data from $\mathrm{TbMnO}_{3}$ crystal 2 and $\mathrm{Sm}_{0.6} \mathrm{Y}_{0.4} \mathrm{MnO}_{3}$ which were grown under argon rather than in air. The loss mechanisms in this temperature interval are evidently sensitive to the oxygen content of the sample.

Figure 12(a) contains fits of equation (1) to representative peaks in $Q^{-1}$ which have $T_{\mathrm{m}}$ near $10 \mathrm{~K}$. In reality there are probably two frequency dependent peaks in the vicinity of 8 and $15 \mathrm{~K}$ but the latter is not readily resolved in data from many of the other resonances. Average values of $E_{\mathrm{a}} / r_{2}(\beta)$ are $\sim 0.003 \mathrm{eV}$ for $\mathrm{TbMnO}_{3}$ crystal 1 and $\mathrm{TbMn}_{0.98} \mathrm{Fe}_{0.02} \mathrm{O}_{3}$, and $\sim 0.004 \mathrm{eV}$ for $\mathrm{TbMnO}_{3}$ crystal 2. Figure 12(b) is an Arrhenius plot of results from fits to different resonance peaks of $\mathrm{TbMnO}_{3}$ crystal 2 and $\mathrm{TbMn}_{0.98} \mathrm{Fe}_{0.02} \mathrm{O}_{3}$. The straight lines fit to the data give values for $E_{\mathrm{a}}$ of $0.0017 \pm 0.0003$ and $0.0021 \pm 0.0007 \mathrm{eV}$ respectively. Corresponding values of $\tau_{\mathrm{o}}$ are $1.4 \times 10^{-8} \mathrm{~s}$ and $1.1 \times 10^{-8} \mathrm{~s}$. From the evidence of overlapping peaks in $Q^{-1}$ it is likely that there are two loss mechanisms with each having $E_{\mathrm{a}}$ in the vicinity of $0.002 \mathrm{eV}$.

\subsection{Comparison with dielectric relaxation}

Figure 13 presents a comparison of representative acoustic loss data, $Q^{-1}$, for $\mathrm{GdMnO}_{3}$ and $\mathrm{TbMn}_{0.98} \mathrm{Fe}_{0.02} \mathrm{O}_{3}$ from 

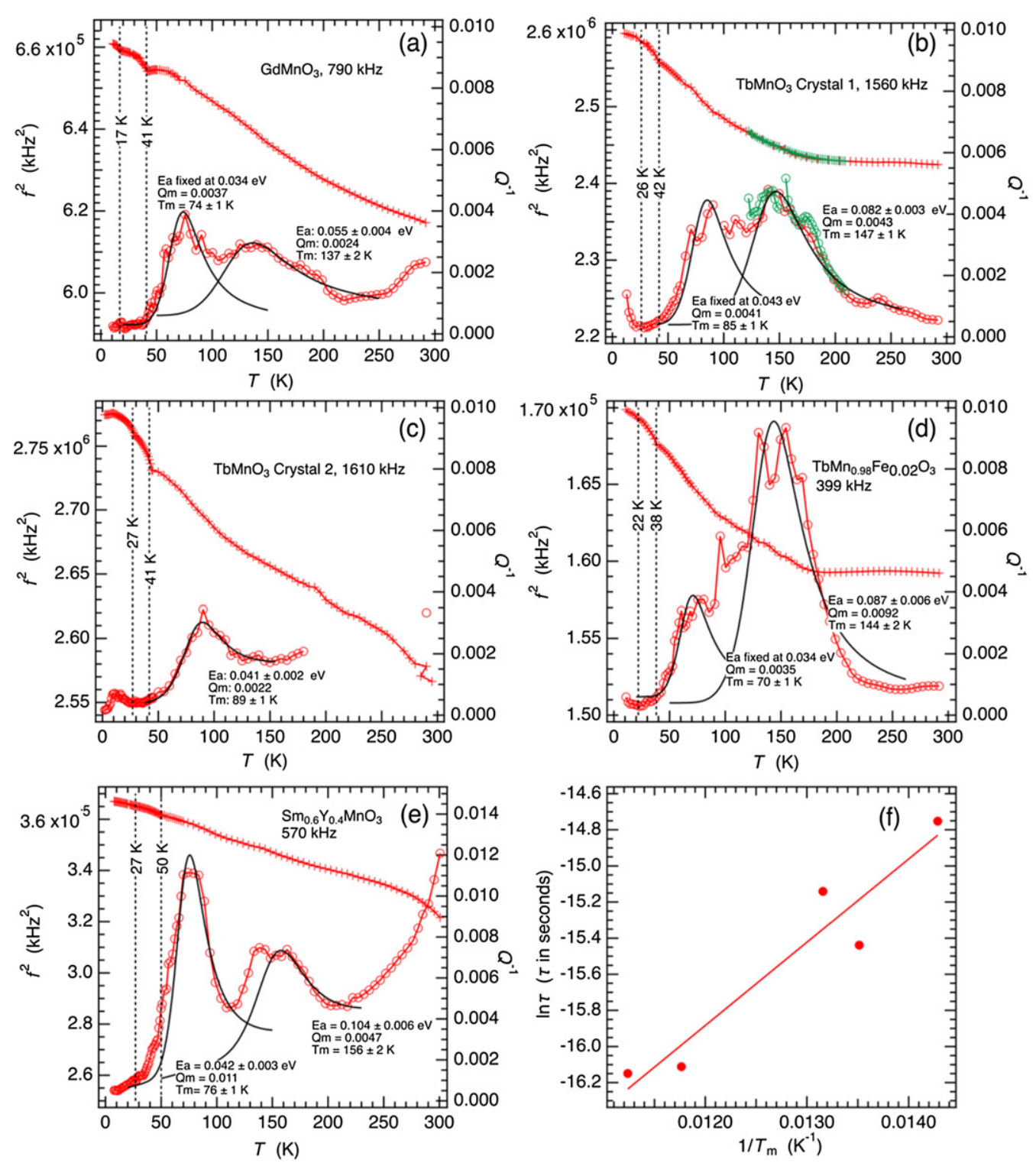

Figure 11. Comparison of the variations of $f^{2}$ and $Q^{-1}$ for representative resonances from the four samples described in the present study (a)-(d) and from $\mathrm{Sm}_{0.6} \mathrm{Y}_{0.4} \mathrm{MnO}_{3}$ [30] (e). Black curves are solutions of equation (1) showing that the data for $Q^{-1}$ can be represented in terms of two loss peaks with $T_{\mathrm{m}} \sim 80, \sim 150 \mathrm{~K}$ and $E_{\mathrm{a}} / r_{2}(\beta) \sim 0.04, \sim 0.09 \mathrm{eV}$, respectively. Constant baselines were assumed in the fitting procedure for (a)-(c) and linear, temperature dependent baselines were assumed for (d) and (e). (f) Is an Arrhenius plot of data for peaks with $T_{\mathrm{m}} \sim 70-90 \mathrm{~K}$ from the fits in (a)-(e). The slope of the straight line fit to the data is $510 \pm 86 \mathrm{~K}$ and the intercept is $-22.1 \pm 1.1$.

this study with dielectric loss, $\tan \delta\left(\mathrm{GdMnO}_{3}\right.$ data from Vilarinho et al [25], for a ceramic sample; $\mathrm{TbMn}_{0.98} \mathrm{Fe}_{0.02} \mathrm{O}_{3}$ unpublished data of A Maia, measured parallel to [001] of a single crystal from the same boule as the crystal used for RUS). The most prominent feature of the $\tan \delta$ data shown for $\mathrm{GdMnO}_{3}$ (figure 13(a)), a frequency-dependent peak in the temperature interval $\sim 200-250 \mathrm{~K}$, does not appear to correlate with any of the data for $Q^{-1}$. Arrhenius treatment of the temperature at which $\omega \tau=1$ gave an activation energy of $0.28 \pm 0.03 \mathrm{eV}$. The pattern is similar for $\mathrm{TbMn}_{0.98} \mathrm{Fe}_{0.02} \mathrm{O}_{3}$, though the peaks are shifted to higher temperatures (figure 13(b)). On the other hand, there is close correlation between $Q^{-1}$ variations and a very much weaker peak in $\tan \delta$ at $\sim 60-70 \mathrm{~K}$ (figures $13(\mathrm{c})$ and (d)). Arrhenius treatment using the condition $\omega \tau=1$ for this peak at $500 \mathrm{kHz}$ and $1 \mathrm{MHz}$ gave $E_{\mathrm{a}}=0.019, \tau_{\mathrm{o}}=7 \times 10^{-9} \mathrm{~s}$ and $0.026 \mathrm{eV}$, $3 \times 10^{-9} \mathrm{~s}$, respectively for $\mathrm{GdMnO}_{3}$ and $\mathrm{TbMn}_{0.98} \mathrm{Fe}_{0.02} \mathrm{O}_{3}$. Ferreira et al [21] obtained $E_{\mathrm{a}}=0.018 \mathrm{eV}$ from their more comprehensive data set for $\mathrm{GdMnO}_{3}$.

Fitting the results for $\tan \delta$ collected as a function of temperature at $500 \mathrm{kHz}$ with the equivalent expression to equation (1) gave $E_{\mathrm{a}} / r_{2}(\beta) \sim 0.016$ and $0.019 \mathrm{eV}$, consistent with $r_{2}(\beta)$ being close to 1 . The value of $E_{\mathrm{a}} / r_{2}(\beta)$ from fitting of the peak in $Q^{-1}$ near $80 \mathrm{~K}$ is $\sim 0.04 \mathrm{eV}$. Although the acoustic data are too noisy to resolve a frequency dependence of the loss peak, this is at least permissive of it being due to a loss mechanism which involves freezing of local electric dipole motion coupled with strain. Values of both $Q^{-1}$ and $\tan \delta$ reduce steeply as $T \rightarrow T_{\mathrm{N} 1}$ for both samples represented in figure 13. There are anomalies 

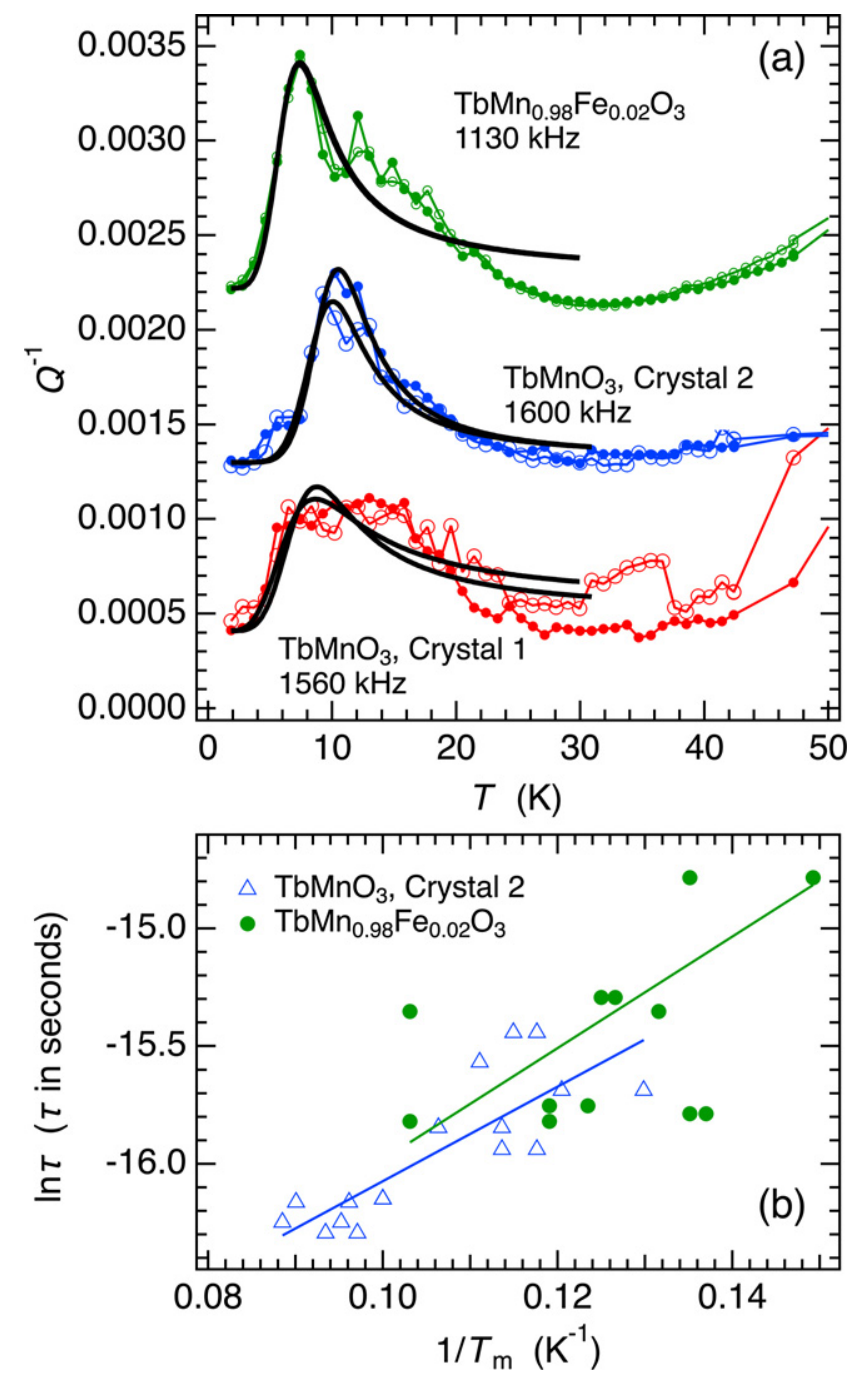

Figure 12. Analysis of loss peaks in the vicinity of $10 \mathrm{~K}$. (a) Fits of equation (1) to $Q^{-1}$ data from representative resonances of the primary RUS spectra. Although a single peak has been fit, there are clearly two loss peaks in the data from $\mathrm{TbMnO}_{3}$ crystal 1 and $\mathrm{TbMn}_{0.98} \mathrm{Fe}_{0.02} \mathrm{O}_{3}$. Open circles = cooling sequence; filled circles = heating sequence. (b) Arrhenius plot of data for $T_{\mathrm{m}}$ from fits of equation (1) to $Q^{-1}$ variations from numerous resonances. The two straight lines fit to the data (more scattered for $\mathrm{TbMn}_{0.98} \mathrm{Fe}_{0.02} \mathrm{O}_{3}$ ) are the same within experimental uncertainty and are consistent with an activation energy of $\sim 0.002 \mathrm{eV}$ for the loss process. $T_{\mathrm{m}}$ values for $\mathrm{TbMnO}_{3}$ crystal 1 are not shown because of scatter due to the overlap of two peaks evident in (a).

in $\tan \delta$ at $T_{\mathrm{N} 2}$, but their form is quite different for $\mathrm{GdMnO}_{3}$ and $\mathrm{TbMn}_{0.98} \mathrm{Fe}_{0.02} \mathrm{O}_{3}$ (figures 13(c) and (d)), implying that the dielectric relaxation mechanism involves only very weak coupling with strain in this case.

The dielectric constant of $\mathrm{TbMnO}_{3}$ shows the same steep frequency-dependent increase with increasing temperature above $\sim 120-150 \mathrm{~K}[18,19,26]$. Cui et al [18] reported activation energies of 0.15 and $0.31 \mathrm{eV}$ for peaks in $\tan \delta$ at $T<160 \mathrm{~K}$, and $T>170 \mathrm{~K}$, respectively, for their polycrystalline sample. The latter is most likely to be due to the same loss process as accounts for the peak in $\tan \delta$ at $\sim 200-250 \mathrm{~K}$ in $\mathrm{GdMnO}_{3}$. The former is perhaps due to the same loss mech- anism as would account for the acoustic loss peak seen at $\sim 150 \mathrm{~K}$ in all four samples.

\subsection{Spontaneous strain}

A formal analysis of spontaneous strains associated with phase transitions in these multiferroic perovskites, based on lattice parameter and high resolution thermal expansion data from the literature, is presented in the appendix A. There are potentially five discrete order parameters, relating to cooperative Jahn-Teller distortions, octahedral tilting, two for magnetic structures based on ordering of $\mathrm{Mn}^{3+}$ and magnetic ordering of $\mathrm{Gd}^{3+} / \mathrm{Tb}^{3+}$, each of which will be coupled to some extent with strain. The combination of octahedral tilting and cooperative Jahn-Teller distortions gives rise to two non-zero shear strains, $e_{4}$ and $e_{\mathrm{t} x}$, with respect to the parent cubic structure [63]. $e_{\mathrm{t} x}$ is a tetragonal strain with its unique axis parallel to the [001] in the Pbnm setting and $e_{4}$ is a shear strain in the plane perpendicular to this, i.e. containing $a$ and $b$ of Pbnm. Values of $e_{4} \sim 10 \%$ and $e_{\mathrm{tx}} \sim-7 \%$ listed in table A1 for $\mathrm{GdMnO}_{3}, \mathrm{TbMnO}_{3}$ and $\mathrm{Sm}_{0.6} \mathrm{Y}_{0.4} \mathrm{MnO}_{3}$ at room temperature are a reflection of the large contribution from the Jahn-Teller distortion of $\mathrm{Mn}^{3+}$. By way of comparison, pure tilting transitions in $\mathrm{GdAlO}_{3}, \mathrm{TbAlO}_{3}$ and $\mathrm{CaTiO}_{3}$ give values of $e_{4} \sim 1 \%$ and $e_{\mathrm{t} x} \sim-0.5 \%$ at room temperature (table A1). The strains at room temperature are also significantly greater than from the combined contributions of tilting and Jahn-Teller distortions in $\mathrm{LaMnO}_{3}, e_{4} \sim 3 \%, e_{\mathrm{t} x} \sim-4 \%$ (table A1). Thus the low temperature magnetic transitions take place in crystals which have a high degree of anisotropic shear strain [1].

Calculating linear strains with respect to the crystallographic axes of the Pbnm structure (table A1) shows that the preferred orientation of magnetic moments in the colinearsinusoidal antiferromagnetic structure and the propagation direction of both the $a b$ - and $b c$-cycloidal magnetic structures ([010]), is parallel to the direction in which the Jahn-Teller distortions produce elongation by $\sim 7 \%$ with respect to the reference cubic structure. Linear strains in the other two directions, $e_{1}$ and $e_{3}$, are both negative (contraction).

High resolution thermal expansion data of Meier [64] (see also $[47,65,66])$, show that spontaneous strains arising from coupling with the magnetic order parameters in $\mathrm{GdMnO}_{3}$ and $\mathrm{TbMnO}_{3}$ are about two orders of magnitude smaller than those due to the combination of tilting and Jahn-Teller distortions. They vary in the range $\pm \sim 0.0002$ and, in combination, give a volume strain, $V_{\mathrm{s}}$, of up to $\sim-0.0004$ (figure A2(b)). Linear strains occur ahead of $T_{\mathrm{N} 1}$ in both $\mathrm{GdMnO}_{3}$ and $\mathrm{TbMnO}_{3}$ and, although the temperature of onset and the magnitudes are not well constrained by the data owing to the need to define baselines for the reference structure (figures A2 and A3), these correlate with a well-defined change in trend of the temperature dependence of phonon-frequencies below $\sim 100 \mathrm{~K}$ [21, 67].

The sign and trend of each of the linear strains $\mathrm{GdMnO}_{3}$ and $\mathrm{TbMnO}_{3}$ (figures A3(b) and (d)) is similar above and below $T_{\mathrm{N} 1}$, suggesting that the local structural and magnetic changes responsible for the precursor effects are closely related to the static colinear-sinusoidal incommensurate magnetic structure. 


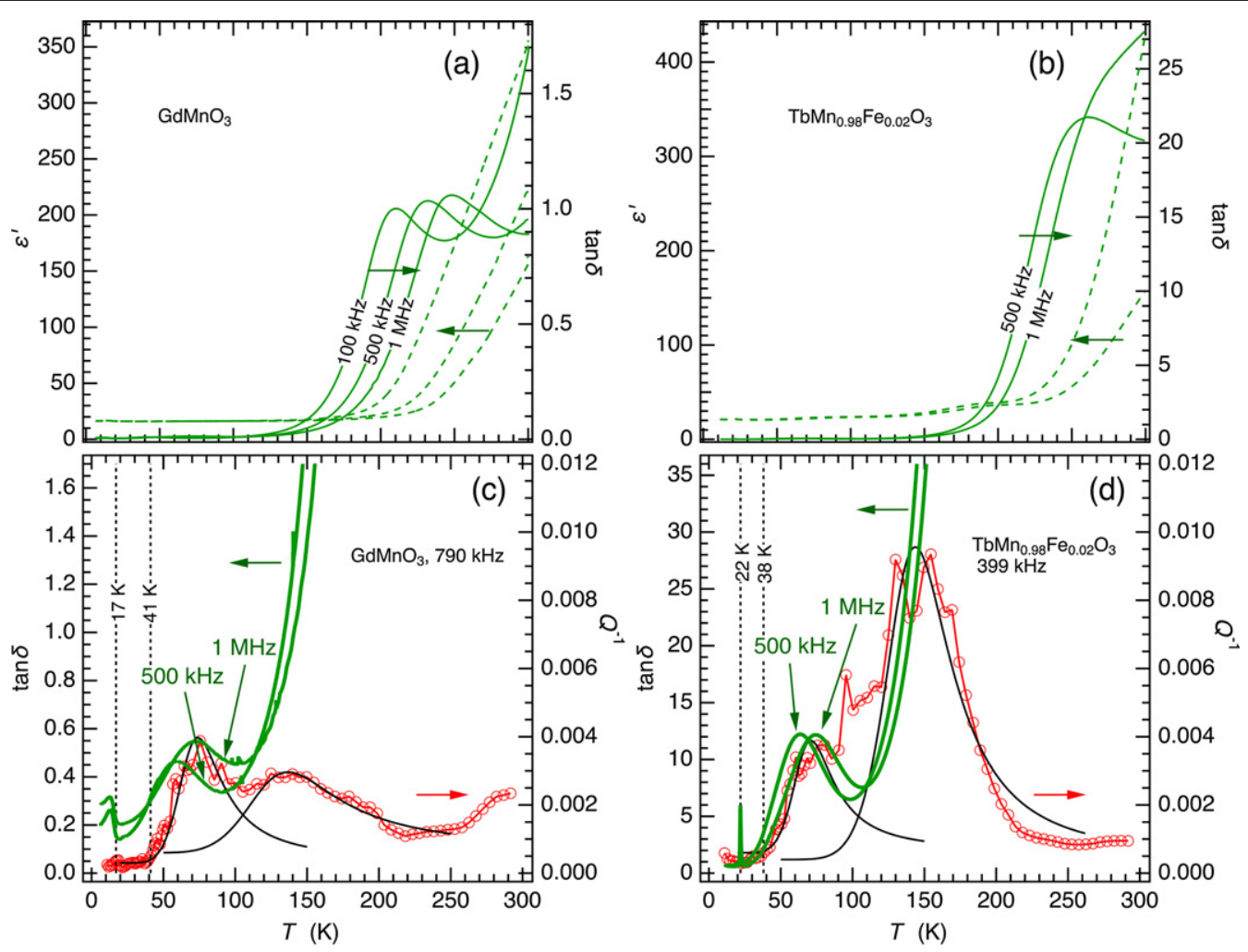

Figure 13. Dielectric spectroscopy data (a) for a ceramic sample of $\mathrm{GdMnO}_{3}$ [25] and (b) for a single crystal of $\mathrm{TbMn}_{0.98} \mathrm{Fe}_{0.02} \mathrm{O}_{3}$, measured parallel to [001] (previously unpublished data of A Maia, MSc Thesis, University of Porto, 2019). The frequency dependent peak in $\tan \delta$ at $\sim 200-300 \mathrm{~K}$ does not obviously correlate with any features in the data for $Q^{-1}$ from this study. (c) and (d) Comparison of $Q^{-1}$ and $\tan \delta$ : a much weaker peak in $\tan \delta$ in the temperature interval $\sim 50-100 \mathrm{~K}$ correlates closely with peaks in $Q^{-1}$. Vertical broken lines mark $T_{\mathrm{N} 1}(41,38 \mathrm{~K})$ and $T_{\mathrm{N} 2}(17,22 \mathrm{~K})$.

Table A1. Spontaneous strains at room temperature determined from lattice parameters given by Cardona Vasquez et al [93] for $\mathrm{GdMnO}_{3}$, by Alonso et al [94] for $\mathrm{TbMnO}_{3}$ and by O'Flynn et al [58] for $\mathrm{Sm}_{0.6} \mathrm{Y}_{0.4} \mathrm{MnO}_{3}$. Room temperature values for the same parameters of $\mathrm{LaMnO}_{3}$ are from Carpenter and Howard [63]. $e_{\mathrm{tx}}$ and $e_{4}$ for $\mathrm{CaTiO}_{3}$ are for pure tilting transitions [92]. Values for $\mathrm{GdAlO}_{3}$ and $\mathrm{TbAlO}_{3}$ were calculated from lattice parameters of Mahana et al [74] and Vasylechko et al [95], respectively. $e_{1}, e_{2}$ and $e_{3}$ are linear strains calculated for a reference system with axes parallel to the crystallographic $a$-, $b$ - and $c$-dimensions, respectively, of the Pbnm cell calculated using the same sets of lattice parameters for the $\mathrm{Gd}-, \mathrm{Tb}$ - and $\mathrm{Sm} / \mathrm{Y}$-manganites.

\begin{tabular}{cccccc}
\hline & $\begin{array}{c}e_{4} \text { (cubic } \\
\text { reference axes) }\end{array}$ & $\begin{array}{c}e_{\mathrm{tx}} \text { (cubic reference } \\
\text { axes) }\end{array}$ & $\begin{array}{c}e_{1} \text { (orthorhombic reference } \\
\text { axes) }\end{array}$ & $\begin{array}{c}e_{2} \text { (orthorhombic } \\
\text { reference axes) }\end{array}$ & $\begin{array}{c}e_{3} \text { (orthorhombic } \\
\text { reference axes) }\end{array}$ \\
\hline $\mathrm{GdMnO}_{3}$ & 0.095 & -0.066 & -0.027 & 0.067 & -0.037 \\
$\mathrm{TbMnO}_{3}$ & 0.10 & -0.070 & -0.029 & 0.072 & -0.039 \\
$\mathrm{Sm}_{0.6} \mathrm{Y}_{0.4} \mathrm{MnO}_{3}$ & 0.095 & -0.068 & -0.027 & & -0.038 \\
$\mathrm{LaMnO}_{3}$ & 0.034 & -0.038 & & \\
$\mathrm{GdAlO}_{3}$ & 0.009 & -0.003 & & \\
$\mathrm{TbAlO}_{3}$ & 0.015 & -0.006 & & \\
$\mathrm{CaTiO}_{3}$ & 0.012 & -0.002 & & & \\
\hline
\end{tabular}

Linear strains coupled to the magnetic order parameter(s) then display differences between the two materials, reflecting differences in details of the evolution of the magnetic structures at lower temperatures. Any additional anomalies in linear strain variations through and below $T_{\mathrm{N} 2}$ are barely detectable in $\mathrm{TbMnO}_{3}$ (figures A3(e)-(g)). In $\mathrm{GdMnO}_{3}$, which has a different orientation of cycloid plane, there is a reversal of the trends of $e_{1}$ and $e_{2}$ with falling temperature (figure A3(b)). The final small adjustments below $T_{\mathrm{R}}$, accompanying ordering on both the $\mathrm{Tb}$ and $\mathrm{Gd}$ sublattices, are positive for $e_{3}$ and negative for $e_{1}$ and $e_{2}$, (figures $\left.\mathrm{A} 3(\mathrm{~b}),(\mathrm{e})-(\mathrm{g})\right)$.
Volume strains are, in effect, an integration of the contribution of all ordering of moments irrespective of the actual ordering scheme and are essentially indistinguishable between $\mathrm{GdMnO}_{3}$ and $\mathrm{TbMnO}_{3}$ (figure A2).

Variations of spontaneous strains due to magnetic ordering in $\mathrm{NdMnO}_{3}$ are shown for comparison in the appendix $\mathrm{A}$, using original data of Meier [64] (and see also [66]). The paramagnetic to A-type antiferromagneic transition at $\sim 85 \mathrm{~K}$, with slight canting to give a weak ferromagnetic moment parallel to [001] [1, 2, 68, 69] is accompanied by linear strains with a temperature dependence which is typical of 
a co-elastic phase transition. There are small contributions from precursor effects, but the magnitudes of the strains that develop below the Néel point are comparable with the combined contributions below and above $\sim 40 \mathrm{~K}$ in $\mathrm{GdMnO}_{3}$ and $\mathrm{TbMnO}_{3}$. Moreover, the onset temperatures for the precursor strains in the latter are more or less the same as in $\mathrm{NdMnO}_{3}$ (compare figures A3(b) and (d), A4). The volume strains for all three materials have closely similar temperature dependences (figures A2(b), A4(b)), and there is only a slight break in slope at $T_{\mathrm{N} 1}$ of $\mathrm{GdMnO}_{3}$ and $\mathrm{TbMnO}_{3}$ in comparison with the sharper and more classical break in slope at the transition point of $\mathrm{NdMnO}_{3}$. The implication is that the total strain coupling is the same for all three materials but that a substantial proportion of this arises from local magnetic ordering ahead of the phase transitions in $\mathrm{GdMnO}_{3}$ and $\mathrm{TbMnO}_{3}$. The small change in strain due to ordering of $\mathrm{Nd}$ moments below $\sim 20 \mathrm{~K}$ in $\mathrm{NdMnO}_{3}$ (figure $\mathrm{A} 4(\mathrm{~b})$ ) is comparable in magnitude with the small additional strains that occur below $T_{\mathrm{R}}$ in $\mathrm{GdMnO}_{3}$ and $\mathrm{TbMnO}_{3}$.

\subsection{Strain/order parameter coupling}

The fact that changes in resonance frequencies at $T_{\mathrm{N} 1}$ and $T_{\mathrm{N} 2}$ described above confirms that coupling of the magnetic order parameters with strain is weak. Lowest order coupling terms of linear strains, $e_{1}, e_{2}, e_{3}$, with the driving order parameter, $M$, for magnetic transitions in an orthorhombic crystal will have the form $\lambda e M^{2}$. These would be expected to give rise to the classic pattern of elastic softening below a second order transition shown first for the displacive transition in $\mathrm{SrTiO}_{3}$ [70]. However, such softening requires that the order parameter can relax on the same time scale as the variations of strain in the acoustic resonance modes. This is seen at the antiferromagnetic ordering transition in $\mathrm{CoF}_{2}$, for example, where the magnitude of the coupled strains is also less than $\sim 0.001$ [71]. Accompanying the elastic anomaly in $\mathrm{CoF}_{2}$ is a classic peak in $Q^{-1}$ due to critical slowing down as the transition point is approached from above and below. The absence of both effects through the magnetic transitions in any of the crystals investigated here implies that relaxation of the magnetic order parameter in response to an induced strain is slower than $\sim 10^{-6}$ s. Evidence of the Debye and dielectric loss peaks at $\sim 80 \mathrm{~K}$ is that switching of local electric dipoles is already reduced to less than this timescale before the transition temperatures are reached. If there is coupling between local magnetic and electric dipole moments, the relaxation time of electric dipoles would provide the rate limiting step for relaxation of the magnetic order parameter.

Coupling of the form $\lambda e^{2} M^{2}$ is always allowed and does not require dynamical relaxation. It will give stiffening or softening in proportion to $M^{2}$, depending on the sign and magnitude of the coupling coefficient, $\lambda$, (as set out in detail in [72], for example). The variation of $f^{2}$ at $T_{\mathrm{N} 1}$ suggests slight stiffening ( $\lambda$ positive). Any anomaly at $T_{\mathrm{N} 2}$ is barely detectable in $\mathrm{TbMnO}_{3}$ (figures A3(d)-(g)), consistent with the colinearsinusoidal to cycloidal magnetic transition being accompanied by changes in the magnitudes of the strain coupling coefficients that are negligibly small. Anomalies in $f^{2}$ at $T_{\mathrm{N} 2}$ and $T_{\mathrm{N} 3}$ in $\mathrm{GdMnO}_{3}$ indicate that there are small differences in the strength of coupling with the magnetic order parameters of the colinear-sinusoidal incommensurate, antiferromagnetic and cycloidal structures.

\subsection{Strain relaxation in the vicinity of $T_{R}$}

Given that there are small changes in $e_{1}, e_{2}$ and $e_{3}$ near $T_{\mathrm{R}}$ for both $\mathrm{GdMnO}_{3}$ and $\mathrm{TbMnO}_{3}$ (figures $\mathrm{A} 3(\mathrm{~b})-(\mathrm{g})$ ), ordering of $\mathrm{Gd}^{3+}$ and $\mathrm{Tb}^{3+}$ moments might to be expected to give rise to closely similar changes of elastic properties. Rounding of the peak in heat capacity (e.g. [42, 45, 52, 55, 73, 74] for $\mathrm{GdMnO}_{3}$ ) is indicative of ordering in a field created by the ordered $\mathrm{Mn}^{3+}$ moments rather than at a discrete phase transition, but there are indeed small anomalies in the temperature dependence of $f^{2}$ close to the expected values of $T_{\mathrm{R}}$ (figures 4 and 8). However, there is a distinct Debye-like peak in $Q^{-1}$ ahead of $T_{\mathrm{R}}$ in the three crystals containing Tb but not in data from the crystal containing Gd. It appears, therefore, that there is a defect pinning or freezing process which is unique to the cycloid structure.

An increase in the imaginary part of the ac magnetic susceptibility of $\mathrm{TbMnO}_{3}$, measured at frequencies of $0.5-3 \mathrm{kHz}$, has also been observed below $\sim 10 \mathrm{~K}$ [29]. The form of this is consistent with there being a peak in magnetic loss below the lowest temperature data point of $5 \mathrm{~K}$. Extrapolation to a measuring frequency of $3 \mathrm{kHz}$ of the straight line fit to the acoustic loss data for crystal 2 in figure 12(b) would give $\omega \tau=1$ at $\sim 3 \mathrm{~K}$. In other words the data are indicative of a magnetoelastic relaxation mechanism, with dynamics constrained by an activation energy of $\sim 0.002 \mathrm{eV}$. The loss mechanism is, as yet, undefined.

Two separate loss peaks in figure 12(a) imply two separate processes with slightly different relaxation times. One possibility for the loss mechanisms relates to freezing of dynamical motion(s) of $\mathrm{Tb}^{3+}$ moments which have some alignment with $\mathrm{Mn}^{3+}$ moments at $15 \mathrm{~K}$ in $\mathrm{TbMnO}_{3}$ [38], i.e. ahead of $T_{\mathrm{R}}$. A second possibility is suggested by low temperature dielectric spectroscopy data between 5 and $16 \mathrm{~K}$ for $\mathrm{DyMnO}_{3}$ which were interpreted as being due to motion of boundaries between $a b$ - and $b c$-cycloid domains on a timescale of $\sim 10^{-7} \mathrm{~s}$ [75]. However, there should not be any shear strain contrast across domain walls of the $b c$-cycloid so that an externally applied shear stress would not be expected to cause them to move. The significant point is that the activation energy barrier is substantially smaller than for the loss process at $\sim 80 \mathrm{~K}$ which is interpreted as being due to coupling of local electric dipoles with polaron-like strain clouds.

\section{Discussion}

An indicative feature of proper ferroelectric, improper ferroelastic transitions arising by ordered displacements of the B-site cation in perovskites is steep softening of the elastic moduli as the transition point is approached from above, as has been observed at RUS frequencies in $\mathrm{BaTiO}_{3}, \mathrm{PbSc}_{0.5} \mathrm{Ta}_{0.5} \mathrm{O}_{3}$, PZN-PT and PIN-PMN-PT, for example [76-79]. The softening is indicative of fluctuations which may be related to a soft 
mode [33]. Crystals containing Tb certainly have some resonance modes which show slight softening (figures 5, 7 and 9) while the crystal of $\mathrm{GdMnO}_{3}$ (figure 3) does not, but the amount of softening is small in comparison. There also appears to be no evidence for a soft mode which might be indicative of an incipient ferroelectric transition. As proposed by Schrettle et al [20], relaxational effects therefore appear to be the dominant feature of the structural dynamics as the low temperature instabilities are approached.

\subsection{Weak coupling of strain with magnetic order parameters in systems with large shear strain due to cooperative Jahn-Teller distortions}

Strain coupling is evidently a significant component of the overall structural and thermodynamic evolution of these systems. The most obvious effect is seen in the relationship between the preferred direction of incommensurate modulations and the orientation of the large strains due to Jahn-Teller distortions and octahedral tilting. The sinusoidal and cycloidal modulations have their propagation directions parallel to [010], the direction in which strain coupled to the Jahn Teller/tilt order parameters is positive $\left(e_{2}\right.$ in table A1). In the other two orthogonal directions, the strains are negative, representing lattice contraction.

Linear strains accompanying the low temperature transitions are very much smaller. They do not have such an obvious orientation relationship with the magnetic structures, but they are clearly dependent on subtle differences in the evolution of the order parameters. On the other hand, the volume strain is essentially an integration of volume reductions associated with ordering of moments at each $\mathrm{Mn}^{3+}$ ion, irrespective of which long-range ordering scheme is adopted. The evolution of $V_{\mathrm{s}}$ is essentially the same for $\mathrm{TbMnO}_{3}$ and $\mathrm{GdMnO}_{3}$ and has the same form as in $\mathrm{NdMnO}_{3}$ (figures $\mathrm{A} 2$ (b) and A4(b)). In $\mathrm{NdMnO}_{3}$ the main transition occurs at $\sim 85 \mathrm{~K}$ and is from a paramagnetic structure to an A-type antiferromagnetic structure (e.g. [1, 2, 68, 69]). It is notable, in particular, that the onset of volume strains in $\mathrm{TbMnO}_{3}$ and $\mathrm{GdMnO}_{3}$ can be detected from $\sim 90 \mathrm{~K}$ (figure A2(b)), indicating that shortrange magnetic ordering develops well above $T_{\mathrm{N} 1}$ in a temperature range that is not dissimilar from that of the long-range ordering in $\mathrm{NdMnO}_{3}$. There are small volume strains associated with ordering of the moments of $\mathrm{Gd}^{3+}, \mathrm{Tb}^{3+}$ and $\mathrm{Nd}^{3+}$, but these are an order of magnitude smaller.

The pattern of volume strains correlates with the pattern of phonon frequencies in the sense that selected modes of $\mathrm{NdMnO}_{3}, \mathrm{TbMnO}_{3}$ and $\mathrm{GdMnO}_{3}$ all show an onset of softening through a similar temperature interval [2, 67, 80].

\subsection{Dynamical precursor state}

Precursor short-range ordering ahead of $T_{\mathrm{N} 1}$ is seen also in other properties. The dc magnetic susceptibility of both polycrystalline and single crystal samples of $\mathrm{GdMnO}_{3}$ has been found to follow the Curie-Weiss law only down to $\sim 80 \mathrm{~K}$ $[29,41,74]$. In $\mathrm{TbMnO}_{3}$ the onset of deviations from Curie-Weiss evolution of the inverse magnetic susceptibility is between $\sim 80 \mathrm{~K}$, measured parallel to [100], and $\sim 200 \mathrm{~K}$, measured parallel to [001] [52]. O'Flynn et al [52] already interpreted this, together with the magnetic field dependence of the heat capacity at $60 \mathrm{~K}$ and a significant precursor effect in the heat capacity (seen also in $\mathrm{Sm}_{0.6} \mathrm{Y}_{0.4} \mathrm{MnO}_{3}$ [58]), in terms of short-range correlations of magnetic moments within the stability field of the paramagnetic structure ahead of $T_{\mathrm{N} 1}$. A similar conclusion was reached for $\mathrm{GdMnO}_{3}$ by Ferreira et al [21] and Vilarinho et al [80] on the basis of their observation of changes in phonon frequencies below $\sim 100 \mathrm{~K}$, again well ahead of the low temperature phase transitions. By way of contrast, the inverse magnetic susceptibility of $\mathrm{GdAlO}_{3}$, which has magnetism arising only from $\mathrm{Gd}^{3+}$, remains linear right down to the Néel point of $\sim 4 \mathrm{~K}$.

The overall implication is that freezing of local ferroelectric dipoles in these manganites is accompanied by the development of a well-developed precursor state involving coupling between local strain and static or dynamical shortrange magnetic order. $\mathrm{TbMnO}_{3}$ also has an anomaly in thermal conductivity which starts to develop below $\sim 150 \mathrm{~K}$ [66].

\subsection{Loss mechanisms within the precursor state}

Acoustic attenuation near $150 \mathrm{~K}$ is most likely related to the increase in electrical conductivity with increasing temperature in all the samples, which accounts also for the steep increase in dielectric loss. In $\mathrm{GdMnO}_{3}$, the activation energy for ac conductivity through the interval $\sim 125-175 \mathrm{~K}$ was reported by $\mathrm{Pal}$ and Murugavel [81] to be $\sim 0.13 \mathrm{eV}$ and the conductivity mechanism was attributed to small polaron hopping. Analysis of a peak in pyrocurrent at $\sim 120 \mathrm{~K}$ by Zhang et al [82] yielded an activation energy of $\sim 0.11 \mathrm{eV}$ and was also attributed to dipole reorientation or release of charge from localised states. This compares with $E_{\mathrm{a}} / r_{2}(\beta)$ values also close to $\sim 0.1 \mathrm{eV}$ from fitting of the peak in $Q^{-1}$ (figure 11). The pattern of loss in this temperature range is also sensitive to the oxygen content of the sample, as seen by the difference between $Q^{-1}$ data above $\sim 120 \mathrm{~K}$ for $\mathrm{TbMnO}_{3}$ crystal 1 (figure 5(b)), which was grown in air, and crystal 2 (figure 7(b)), which was grown under argon.

The loss peak near $80 \mathrm{~K}$ appears to be unique to orthorhombic manganite perovskites, as it was not observed in RUS measurements of Pbnm oxide perovskites that do not display magnetism, ferroelectricity or cooperative Jahn-Teller distortions, such $\mathrm{CaTiO}_{3}$ [83] and $\mathrm{BaCeO}_{3}$ [84]. It was also not observed in the multiferroic hexagonal manganite $\mathrm{YMnO}_{3}$ [31] which has an antiferromagnetic ordering transition at $\sim 75 \mathrm{~K}$. In view of the close relationship between the acoustic and dielectric properties shown in figure 13, there is little doubt that the loss mechanism involves freezing of electric dipoles with local strain coupling. The loss parameters from fitting the data in terms of a thermally activated process in figure 11(f), $\tau_{\mathrm{o}} \sim 10^{-10} \mathrm{~s}, E_{\mathrm{a}} \sim 0.04 \mathrm{eV}$, provide a measure of the dynamics of this mechanism.

Activation energies of $\sim 0.07 \mathrm{eV}$ appear to be characteristic of polaronic relaxation in perovskites more generally [85]. This view is reinforced by comparison with the dielectric loss patterns in ferroelectric or incipient ferroelectrics which do not have magnetic transitions. For example, the incipient ferroelectric $\mathrm{KTaO}_{3}$ remains cubic down to the lowest measuring 
temperatures but has an acoustic loss peak at $\sim 60 \mathrm{~K}$ with $E_{\mathrm{a}} \geqslant \sim 0.09 \mathrm{eV}$, from a resonance with frequency near $740 \mathrm{kHz}$ [86]. There is also a frequency dependent dielectric loss peak in the temperature interval $\sim 40-60 \mathrm{~K}$ with $E_{\mathrm{a}} \sim 0.04 \mathrm{eV}$ [87], which presumably has the same origin.

Precursor strains which occur in the same temperature interval as these Debye-like freezing processes have the same sign as the strains which continue to develop below $T_{\mathrm{N} 1}$ in $\mathrm{GdMnO}_{3}$ and $\mathrm{TbMnO}_{3}$ and below $T_{\mathrm{N} 2}$ in $\mathrm{TbMnO}_{3}$ (figures $\mathrm{A} 3(\mathrm{~b})$ and (d)). On this basis, it appears that the local dynamical structural state is likely to be related to the configuration of long-range magnetic ordering in the colinear-sinusoidal and cycloidal structures. It should be noted, however, that Pal et al [27] reported activation energies of $\sim 0.03-0.04 \mathrm{eV}$ obtained by analysis of a broad pyrocurrent signal at $\sim 50 \mathrm{~K}$ and a dielectric loss peak in $\mathrm{GdMnO}_{3}$, which they suggested could be extrinsic due to defect dipoles associated with oxygen nonstoichiometry and mixed valence of $\mathrm{Mn}$.

\subsection{Frustration}

The importance of frustration effects, arising from ferromagnetic nearest-neighbour and antiferromagnetic nextnearest-neighbour interactions, in promoting the stability of magnetoelectric structures in $R \mathrm{MnO}_{3}$ perovskites was emphasised from the start by Kimura et al [1]. Competition between different static magnetic ordering schemes results in progressive suppression of the A-type antiferromagnetic structure in the sequence La-, Pr-, Nd-, Sm-, Eu-, Gd-, $\mathrm{TbMnO}_{3}$ associated with increasing lattice distortions that arise from the reductions in ionic radius. It now appears that suppression of long-range ordering in $\mathrm{GdMnO}_{3}$ and $\mathrm{TbMnO}_{3}$ is mitigated by local dynamical ordering which enhances the stability of the paramagnetic structure below $\sim 80-100 \mathrm{~K}$. The more complex incommensurate and cycloidal structures only develop once the thermally activated fluctuations revealed by the patterns of acoustic and dielectric loss have frozen out. These dynamical effects involve electric dipoles which have a relative strong coupling with strain. In addition, however, there must be contributions from weaker coupling of magnetic moments with strain which freeze out within the stability field of the cycloidal structure. Evidence of these is provided by the acoustic loss peaks near $10 \mathrm{~K}$ in the RUS data from $\mathrm{TbMnO}_{3}$ (figure 12).

Frustration effects could be modified by competing strain fields in doped crystals. Replacing $2 \%$ of $\mathrm{Mn}^{3+}$ by $\mathrm{Fe}^{3+}$ is not likely to affect the magnetic ordering but, by comparison with La substitution in $\mathrm{PrAlO}_{3}$ [88], corresponds almost exactly with the substitution limit at which strain fields around one dopant cation would start to overlap with the strain fields around nearby dopant cations and thereby modify the properties of the whole crystal. $f^{2}$ for several resonance modes from $\mathrm{TbMn}_{0.98} \mathrm{Fe}_{0.02} \mathrm{O}_{3}$ (figure 9) show slight softening with falling temperature but there is no evidence of any of the steep softening which would be expected if there was any marked tendency to stabilise a more typical ferroelectric structure, such as in $\mathrm{BaTiO}_{3}$ for example. The origin of the softening therefore is more likely to be a slight adjustment to the coupling of small strains with the magnetic order parameter(s).

\subsection{Implications for thin films and multiferroic domain walls}

Coupling between strain and magnetic or electric dipoles is extensively exploited in thin film applications since the substrate allows the functional properties to be manipulated through the choice of imposed strain fields. Of particular interest in the present context is the fact that thin films of $\mathrm{TbMnO}_{3}$ and $\mathrm{GdMnO}_{3}$ contain abundant self-organised twin domains (e.g. $[9,10,15,89,90])$. While it might not be possible to switch these domains by any external field because of their attachment to the substrate, they are in effect ferroelastic twins due to lowering of symmetry from cubic or tetragonal to orthorhombic. Because the tilt, Jahn-Teller and magnetic order parameters all couple with strain, it is inevitable that steep strain gradients through ferroelastic twin walls will cause the magnetic and ferroelectric properties of the twin walls to differ from those of the twin domains. For example, Daumont et al [90] have proposed that a high density of twin walls in a thin film of $\mathrm{TbMnO}_{3}$ on $\mathrm{SrTiO}_{3}$ might contribute to ferromagnetism which is not seen in bulk samples.

There do not appear to be reports of ferroelastic twinning in single crystals but if it was possible to grow crystals containing multiple ferroelastic domains walls, such as have been observed in $\mathrm{LaMnO}_{3}$ for example (see figure 1(a) of Dechamps et al [91]), they would be expected to display quite different magnetoelectric properties and should lead to new possibilities for engineering of functional nanostructures. The mobility of ferroelastic and multiferroic domain walls in such crystals will be constrained significantly by the polaronic freezing effects identified here but, if they are sufficiently broadened by the influence of their magnetic component, they might become able to ride over the strain fields of local pinning points. In $\mathrm{DyMnO}_{3}$, for example, domain walls between $a b$ - and $b c$ cycloid domains appear to be mobile on a timescale of less than $\sim 10^{-6}$ s down to at least $5 \mathrm{~K}$ [75].

\section{Conclusion}

Measurements of elastic and anelastic properties have revealed a well-defined temperature interval ahead of the magnetoelectric phase transitions in $\mathrm{GdMnO}_{3}$ and $\mathrm{TbMnO}_{3}$ where local, dynamic correlations of electric and magnetic polarisation couple with strain on a time scale of $\sim 10^{-6}$ s. Relaxation times reduce with falling temperature in a manner that is consistent with a rate determining step which depends on the mobility of polaron-type strain clouds, such that the magnetically ordered structures become stable only after these have become effectively immobile. Stabilisation of the paramagnetic structure by dynamical, short-range ordering local magnetic and ferroelectric moments, at the expense of the homogeneous A-type antiferromagnetic structure, is likely to be a significant factor in determining the form of the phase diagram in which the transition temperature of the first magnetic transition reduces from La through to $\mathrm{Gd}$ and then increases from Dy to Ho. Magnetoelastic loss peaks at lower temperatures demonstrate, also, that aspects of the magnetoelectric structure remain mobile down to at least $\sim 5 \mathrm{~K}$. 


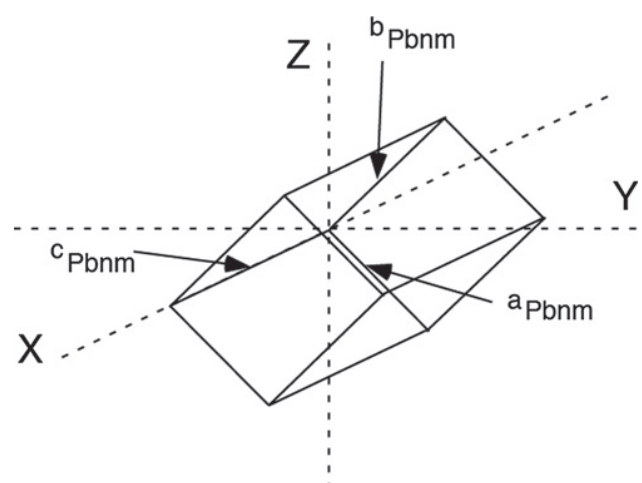

Figure A1. Reference system used to calculate values of shear strains $e_{\mathrm{t} x}$ and $e_{4}$. In both $\mathrm{TbMnO}_{3}$ and $\mathrm{GdMnO}_{3}$ magnetic moments of the colinear-sinusoidal incommensurate antiferromagnetic structure are parallel and antiparallel to $b_{P b n m}$. The repeat direction of the cycloidal structure is also parallel to $b_{P b n m}$. In $\mathrm{TbMnO}_{3}$ the cycloid is within the $b c$-plane and the electric polarisation is parallel to $c_{P b n m}$. In $\mathrm{GdMnO}_{3}$ the cycloid is within the $a b$-plane, the electric polarisation is parallel to $a_{P b n m}$ and the weak ferromagnetic moment arising from canting is parallel to $c_{P b n m}$.

\section{Acknowledgments}

We thank Professor T Lorenz, University of Cologne, for constructive criticisms of the manuscript. This work was funded by EPSRC Grant No. EP/P024904/1 (UK), and Projects Nos. NORTE-01-0145-FEDER-022096, UID/NAN/50024/2019, SK-PT-2015-0030 and VEGA No. 2/0137/19 (Slovakia). RUS facilities were established through Grants from the Natural Environment Research Council (Grants Nos. NE/B505738/1 and NE/F017081/1) and the Engineering and Physical Sciences Research Council (Grant No. EP/I036079/1) to MAC. The work at the University of Warwick was supported by EPSRC, UK through Grant No. EP/T005963/1. Funding through the NTNU Onsager Fellowship programme is also acknowledged.

\section{Appendix A. Strain analysis}

Figure A1 shows the reference system used to calculate values of shear strains $e_{\mathrm{t} x}$ and $e_{4}$ from lattice parameter data at room temperature. These symmetry-adapted strains correspond to a tetragonal shear strain with its unique axis parallel to [001] in the Pbnm setting, and a shear strain in the $a b$-plane. $e_{\mathrm{t} x}$ is given by $(1 / \sqrt{ } 3)\left(2 e_{1}-e_{2}-e_{3}\right)$, where $e_{1}$ is the strain parallel to $X$, $e_{2}$ is the strain parallel to $Y$ and $e_{3}$ is the strain parallel to $Z$, as defined with respect to a cubic reference structure. Values of the lattice parameter for the reference cubic structure, $a_{0}$, have been taken to be $\left(a_{P b n m} \cdot b_{P b n m} \cdot c_{P b n m} / 4\right)^{1 / 3}$. The full set of equations is given in Carpenter et al [92]. Room temperature data for $e_{4}$ and $e_{\mathrm{t} x}$ in $\mathrm{GdMnO}_{3}, \mathrm{TbMnO}_{3}$ and $\mathrm{Sm}_{0.6} \mathrm{Y}_{0.4} \mathrm{MnO}_{3}$, calculated using lattice parameter taken from the literature, are listed in table A1. Data for $\mathrm{LaMnO}_{3}, \mathrm{GdAlO}_{3}, \mathrm{TbAlO}_{3}$ and $\mathrm{CaTiO}_{3}$ are included for comparison. For strains determined with respect to an orthorhombic reference structure: $e_{1}=\left(\left(a_{P b n m} / \sqrt{ } 2\right)-a_{\mathrm{o}}\right) / a_{\mathrm{o}}, e_{2}=\left(\left(b_{P b n m} / \sqrt{ } 2\right)-a_{\mathrm{o}}\right) / a_{\mathrm{o}}$, $e_{3}=\left(\left(c_{\text {Pbnm }} / 2\right)-a_{\mathrm{o}}\right) / a_{\mathrm{o}}$.
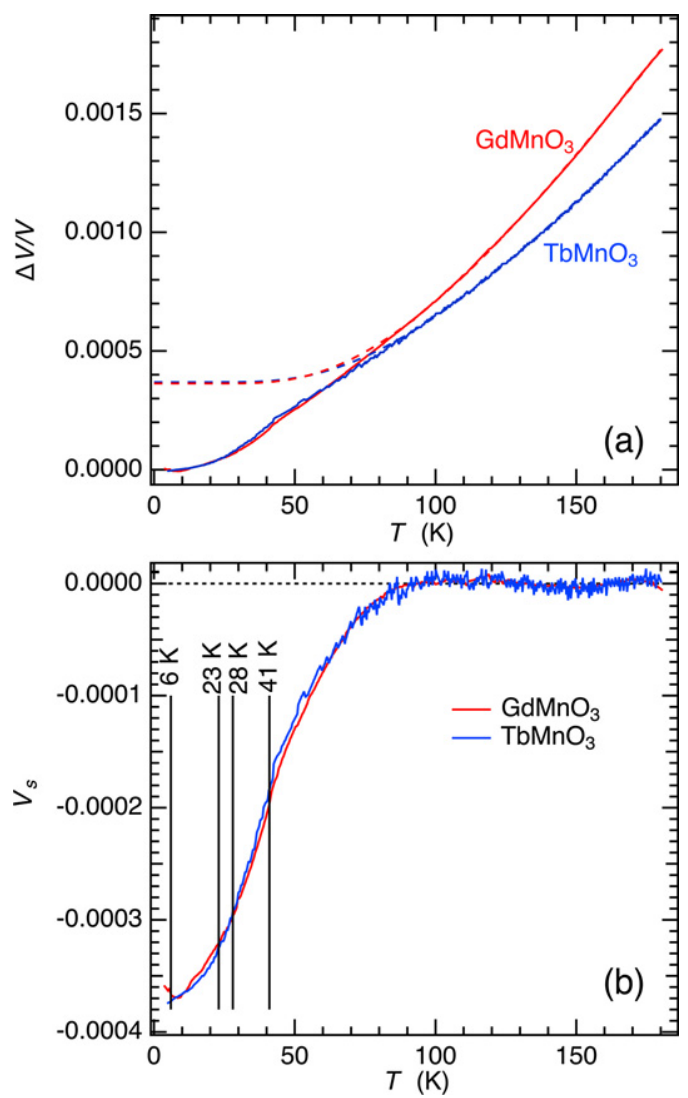

Figure A2. (a) High resolution volume expansion for $\mathrm{GdMnO}_{3}$ and $\mathrm{TbMnO}_{3}$ obtained by summation of data for the three linear expansion directions reported in Meier [64]. Dashed lines are baselines, representing volume expansion of the high symmetry structure, obtained by fitting equation (A1) to data in the temperature interval $85-181 \mathrm{~K}$. (b) Variations of volume strain, $V_{\mathrm{s}}$, given by the difference between the measured values and baseline values of $\Delta V / V$ shown in (a).

Linear thermal expansion data from Meier [64] (and see also $[47,65,66])$ are reproduced in figures A2 and A3 in order to illustrate the form and magnitude of spontaneous strains which arise by coupling with the driving order parameters at the low temperature transitions in $\mathrm{GdMnO}_{3}$ $\left(T_{\mathrm{N} 1} \approx 41 \mathrm{~K}, T_{\mathrm{N} 2} \approx 23 \mathrm{~K}, T_{\mathrm{R}} \approx 6 \mathrm{~K}\right)$ and $\mathrm{TbMnO}_{3}$ $\left(T_{\mathrm{N} 1} \approx 41.5 \mathrm{~K}, T_{\mathrm{N} 2} \approx 28 \mathrm{~K}, T_{\mathrm{R}} \approx 7 \mathrm{~K}\right)$. The raw data are given in the form $\Delta a / a, \Delta b / b, \Delta c / c$ where $\Delta a, \Delta b$ and $\Delta c$ are changes in linear dimensions of a single crystal measured parallel to the crystallographic $x$-, $y$ - and $z$-axes of space group $P b n m$, and $a, b, c$ are the lattice parameters at the lowest temperature at which measurements were made. The change in volume, $\Delta V / V$ is given by $\Delta a / a+\Delta b / b+\Delta c / c$. In this form, the three linear spontaneous strains, $e_{1}, e_{2}, e_{3}$, and volume strain, $V_{\mathrm{s}}$, are given by the difference between observed values and values of a baseline extrapolated from fits to data above the transition temperatures. A convenient expression to represent the baselines, taking account of the requirement that they have zero slope as $T \rightarrow 0 \mathrm{~K}$, is

$$
\text { baseline value }=A_{\mathrm{o}}+A_{1} \Theta_{\mathrm{s}} \operatorname{coth}\left(\frac{\Theta_{\mathrm{s}}}{T}\right) \text {, }
$$



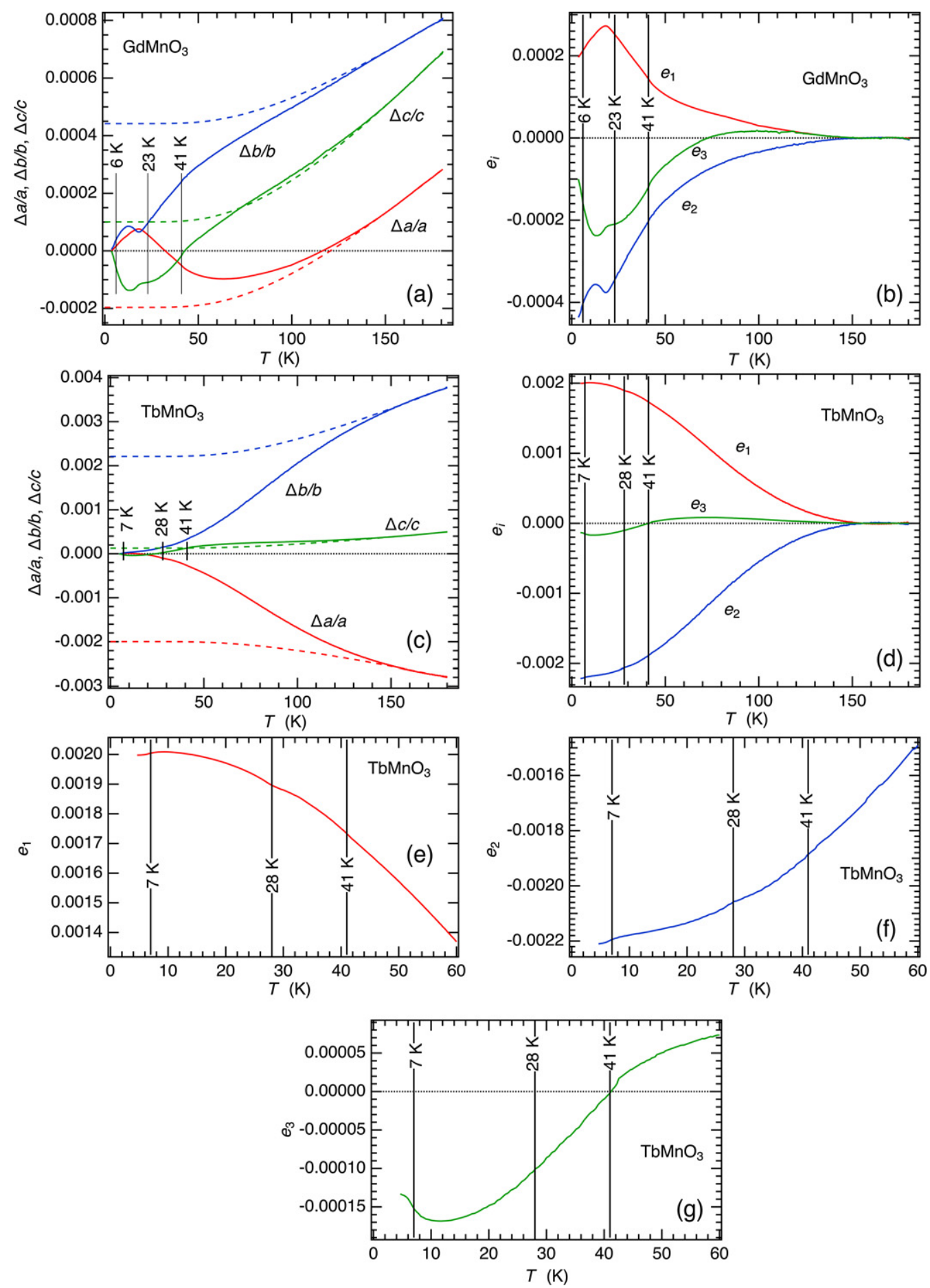

Figure A3. (a) and (c) High resolution linear thermal expansion data for $\mathrm{GdMnO}_{3}$ and $\mathrm{TbMnO}_{3}$ reported in Meier [64]. Dashed lines are baselines, representing linear expansion of the high symmetry structure, obtained by fitting equation (A1) to data in the temperature interval $149-181 \mathrm{~K}$ with $\Theta_{\mathrm{s}}$ fixed at $137 \mathrm{~K}$ for $\mathrm{GdMnO}_{3}$ and $135 \mathrm{~K}$ for $\mathrm{TbMnO}_{3}$. (b) and (d) Variations of the spontaneous strains, $e_{1}, e_{2}, e_{3}$ given by the difference between measured values and baseline values of $\Delta a / a, \Delta b / b, \Delta c / c$. (e)-(g) Expanded views of the linear strains for $\mathrm{TbMnO}_{3}$ at the lowest temperatures.

where $A_{\mathrm{o}}$ and $A_{1}$ are constants, $T$ is temperature and $\Theta_{\mathrm{s}}$ is a saturation temperature (following [96-100]). Fitting this expression to the unit cell volume data of Agostinho Moreira et al [101] over a wider temperature interval up to $250 \mathrm{~K}$ and excluding the influence of the phase transitions gave $\Theta_{\mathrm{s}}$ $\approx 150 \mathrm{~K}$, which is typical of values in the range $\sim 100-150 \mathrm{~K}$ obtained for the evolution of thermal expansion in other perovskites as $T \rightarrow 0 \mathrm{~K}$ (e.g. [102-104]).

Fitting equation (A1) to the volume expansion data in the temperature interval $85-181 \mathrm{~K}$ gave $\Theta_{\mathrm{s}}=137 \mathrm{~K}$ for $\mathrm{GdMnO}_{3}$ and $\Theta_{\mathrm{s}}=135 \mathrm{~K}$ for $\mathrm{TbMnO}_{3}$. These baselines are shown as dashed lines in figure A2(a) and are 


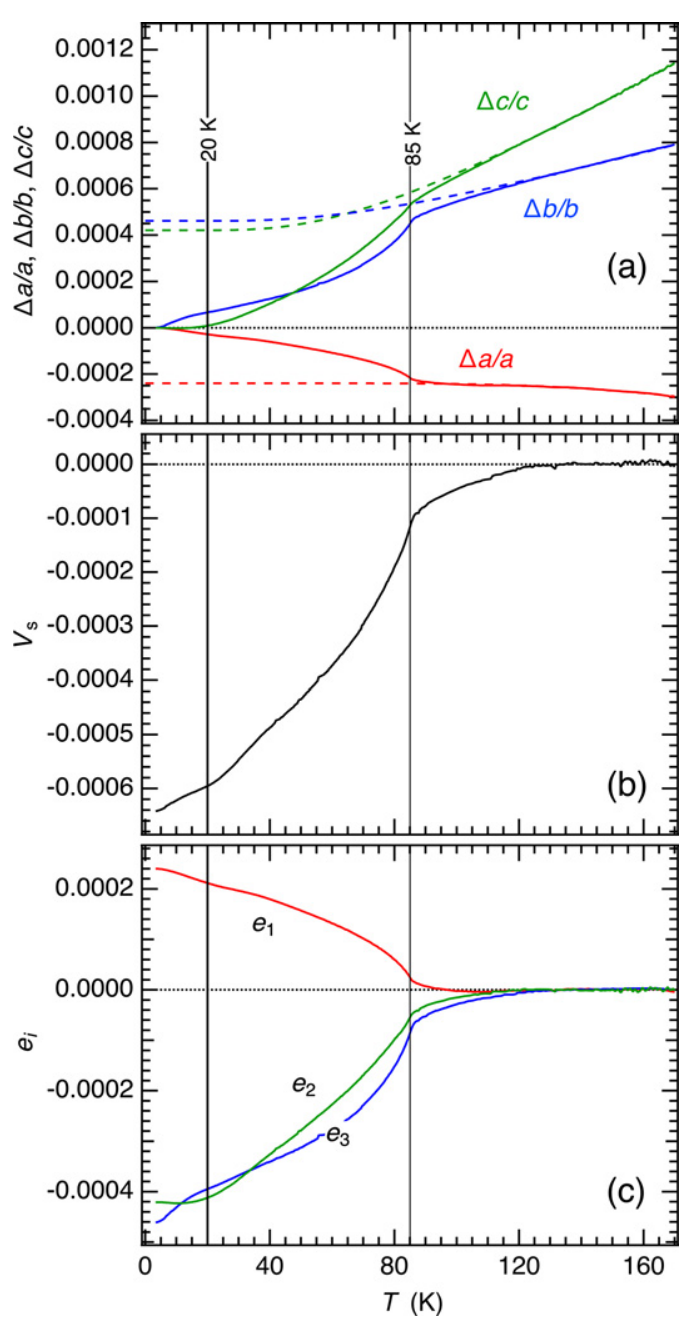

Figure A4. (a) High resolution linear thermal expansion data for $\mathrm{NdMnO}_{3}$ reported in Meier [64] and Berggold et al [66]. Dashed lines are baselines, representing linear expansion of the high symmetry structure, obtained by first fitting equation (A1) to data in the temperature interval $123-190 \mathrm{~K}$ for $\Delta c / c$ which gave $\Theta_{\mathrm{s}}=$ 104.7 K. This value of $\Theta_{\mathrm{s}}$ was fixed when fitting to the data for $\Delta b / b$ in the same temperature interval. The baseline for $\Delta a / a$ was fit to data in the temperature interval $120-170 \mathrm{~K}$ and had $\Theta_{\mathrm{s}}=369 \mathrm{~K}$.

similar to equivalent extrapolations of Raman mode frequencies reported for $\mathrm{TbMnO}_{3}$ by Mansouri et al [67] and for $\mathrm{GdMnO}_{3}$ by Vilarinho et al [80]. Volume strains, $V_{\mathrm{s}}$, given by the difference between baseline values and observed values are shown in figure $\mathrm{A} 2(\mathrm{~b}) . V_{\mathrm{s}}$ variations associated with the low temperature transitions are indistinguishable for both materials and appear to consist of two parts. A precursor effect amounts to $\sim-0.0002$ below $\sim 80 \mathrm{~K}$ and a further change of $\sim-0.0001$ occurs below $T_{\mathrm{N} 1}$. There is no obvious anomaly at $T_{\mathrm{N} 2}$ or $T_{\mathrm{R}}$. The negative sign of $V_{\mathrm{s}}$ is consistent with positive values of $\mathrm{d} T_{\mathrm{N}} / \mathrm{d} P$ for all three transitions in $\mathrm{TbMnO}_{3}$ [105].

Repeating the baseline fitting procedure for each of $\Delta a / a$, $\Delta b / b, \Delta c / c$ in the temperature interval $149-181 \mathrm{~K}$, keeping the value of $\Theta_{\mathrm{s}}$ fixed at $137 \mathrm{~K}$ for $\mathrm{GdMnO}_{3}$ and $135 \mathrm{~K}$ for $\mathrm{TbMnO}_{3}$, gave the dashed lines in figures $\mathrm{A} 3(\mathrm{a})$ and (c). Values of linear strains $e_{1}, e_{2}$ and $e_{3}$ due to coupling with the order parameters were then obtained as the difference between the measured and baseline values. There are again precursor effects but variations below $\sim 41 \mathrm{~K}$ amount to only up to $\pm \sim 0.0002$. The magnitude of the precursor effects is highly sensitive to the choice of baseline, but the trend is the same in both $\mathrm{GdMnO}_{3}$ and $\mathrm{TbMnO}_{3}$. There is a difference in magnitude by a factor of 10 between $\mathrm{GdMnO}_{3}$ and $\mathrm{TbMnO}_{3}$, but $e_{1}$ and $e_{2}$ are positive and negative, respectively. $e_{3}$ values remain much smaller, indicating that the principal strain is shearing in the $a b$-plane.

All three linear strains maintain the same trend through $T_{\mathrm{N} 1}$, implying that the dynamical magnetostructural effects above $T_{\mathrm{N} 1}$ are closely related to the static magnetisation of the incommensurate magnetic structure down to $T_{\mathrm{N} 2}$. This has magnetic moments aligned parallel to [010] in the Pbnm setting. The same trends continue below $T_{\mathrm{N} 2}$ in $\mathrm{TbMnO}_{3}$ (figures $\mathrm{A} 3(\mathrm{~d})-(\mathrm{g})$ ) but show small reversals in $\mathrm{GdMnO}_{3}$ (figure $\mathrm{A} 3(\mathrm{~b})$ ). The difference is presumably due to coupling of strains with the cycloidal magnetic ordering in the $a b$-plane of $\mathrm{GdMnO}_{3}$ as opposed to the $b c$-plane of $\mathrm{TbMnO}_{3}$. Electric polarisation is along [100] in the $a b$-cycloid and along [001] in the $b c$-cycloid. There are additional very small changes in linear strains below the temperatures, $T_{\mathrm{R}}$, at which magnetic ordering on $\mathrm{Tb}$ and $\mathrm{Gd}$ contributes to the overall magnetically ordered structure.

These patterns of strain evolution may be contrasted with the strains which accompany magnetic ordering in $\mathrm{NdMnO}_{3}$. The transition in this case occurs at $\sim 85 \mathrm{~K}$ and is from a paramagnetic structure to an A-type antiferromagnetic structure (e.g. $[1,2,68,69])$. Canting of the moments results in weak ferromagnetism with a net moment aligned parallel to [001] [69, 106], though Chatterji et al [68] had proposed that the canting only starts at lower temperatures. Ordering of the moments at $\mathrm{Nd}$ occurs below $\sim 20 \mathrm{~K}[68,69]$. The net ferromagnetic moment due to $\mathrm{Nd}^{3+}$ ordering in the low temperature structure is aligned in the opposite $z$-direction from the moment due to the prior canting of $\mathrm{Mn}^{3+}$ and causes the $\mathrm{Mn}$-canting angle to rotate so that the direction of both becomes the same [69].

Figure A4 contains results from analysis of high resolution linear thermal expansion data for $\mathrm{NdMnO}_{3}$ from Meier [64] (and see also [66]). The primary data are reproduced in figure A4(a). Dashed lines represent baselines from fitting equation (A1) to data above $T_{\mathrm{N} 1}$, in order to estimate the values for $e_{1}, e_{2}, e_{3}$ and $V_{\mathrm{s}}$ (calculated here as $V_{\mathrm{s}}=e_{1}+e_{2}+e_{3}$ ) shown in figures $\mathrm{A} 4(\mathrm{~b})$ and (c). Notwithstanding uncertainties arising from the choice of baselines, maximum values of the strains are closely similar to those obtained for $\mathrm{TbMnO}_{3}$ and $\mathrm{GdMnO}_{3}$. However, in contrast with the large contribution of precursor strains below $\sim 100-150 \mathrm{~K}$ and the small breaks in slope at $T_{\mathrm{N} 1}$ seen for $\mathrm{TbMnO}_{3}$ and $\mathrm{GdMnO}_{3}$, the antiferromagnetic ordering transition in $\mathrm{NdMnO}_{3}$ is marked by a clear break in slope and only small precursor effects. The smoother variations for $\mathrm{TbMnO}_{3}$ are evident also in lattice parameter data reported by Blasco et al [107] for the temperature interval $\sim 2-320 \mathrm{~K}$. The more classical pattern of non-symmetry breaking strains accompanying a co-elastic phase transition (no symmetry breaking shear strain, coupling of the form $\lambda e M^{2}$ giving $e \propto M^{2}$ ) shown 
by $\mathrm{NdMnO}_{3}$ is confirmed by the lattice parameter data of Chatterji et al [68]. There is a further break in slope of the strains in figure 4 at $\sim 21 \mathrm{~K}$, particularly in $V_{\mathrm{s}}$, consistent with the temperature reported by Chatterji et al [68] for ordering of moments of $\mathrm{Nd}$.

In summary, spontaneous strains arising by coupling with the magnetic order parameters are very much smaller than those which would typically arise by coupling with tilt or Jahn-Teller order parameters. The predominant effects in $\mathrm{TbMnO}_{3}$ and $\mathrm{GdMnO}_{3}$, both with respect to linear strains and the combined volume strain, are the precursor contributions ahead of $T_{\mathrm{N} 1}$ and the small changes of slope associated with the magnetic transition at $T_{\mathrm{N} 1}$. Subsequent changes due to the development of the cycloid and associated electric polarisation are even smaller, by comparison. The sign and evolution of each of the linear strains are similar to those below $T_{\mathrm{N} 1}$, consistent with the precursor effects being due to coupling with a dynamically ordered structure which is locally similar to the long-range incommensurate magnetic structure. An important contrast with $\mathrm{NdMnO}_{3}$ is that the latter shows classical variations in strain associated with the discrete antiferromagnetic ordering transition, i.e. a small precursor effect followed by a significant break in slope at the transition point.

\section{ORCID iDs}

M A Carpenter (D) https://orcid.org/0000-0003-2855-0007

D Pesquera (D) https://orcid.org/0000-0003-0681-3371

N Mufti (D) https://orcid.org/0000-0002-8260-8495

A A Nugroho (D) https://orcid.org/0000-0002-1785-4008

M Mihalik Jr (D) https://orcid.org/0000-0002-5316-0089

M Mihalik (D) https://orcid.org/0000-0002-5442-9414

J Agostinho Moreira (D) https://orcid.org/0000-0003-46597503

D Meier (D) https://orcid.org/0000-0002-8623-6705

\section{References}

[1] Kimura T, Ishihara S, Shintani H, Arima T, Takahashi K T, Ishizaka K and Tokura Y 2003 Phys. Rev. B 68 060403(R)

[2] Goto T, Kimura T, Lawes G, Ramirez A P and Tokura Y 2004 Phys. Rev. Lett. 92257201

[3] Kimura T, Lawes G, Goto T, Tokura Y and Ramirez A P 2005 Phys. Rev. B 71224425

[4] Goto T, Yamasaki Y, Watanabe H, Kimura T and Tokura Y 2005 Phys. Rev. B 72 220403(R)

[5] Kirby B J, Kan D, Luykx A, Murakami M, Kundaliya D and Takeuchi I 2009 J. Appl. Phys. 10507 D917

[6] Marti X, Skumryev V, Ferrater C, García-Cuenca M V, Varela M, Sánchez F and Fontcuberta J 2010 Appl. Phys. Lett. 96 222505

[7] Cui Y, Tian Y, Shan A, Chen C and Wang R 2012 Appl. Phys. Lett. 101122406

[8] Romaguera-Barcelay Y, Agostinho Moreira J, Almeida A, Tavares P B and Pérez de la Cruz J 2014 Thin Solid Films 564 419-25

[9] Li X et al 2014 Sci. Rep. 47019

[10] Farokhipoor S et al 2014 Nature 515 379-83

[11] Lu C and Liu J-M 2016 J. Mater. 2 213-24

[12] Fiebig M 2005 J. Phys. D: Appl. Phys. 38 R123
[13] Catalan G, Seidel J, Ramesh R and Scott J F 2012 Rev. Mod. Phys. 84119

[14] Meier D 2015 J. Phys.: Condens. Matter. 27463003

[15] Matsubara M et al 2015 Science 348 1112-5

[16] Fiebig M, Lottermoser T, Meier D and Trassin M 2016 Nat. Rev. Mater. 116046

[17] Noda K, Nakamura S, Nagayama J and Kuwahara H 2005 J. Appl. Phys. $9710 \mathrm{C} 103$

[18] Cui Y, Zhang L, Xie G and Wang R 2006 Solid State Commun. 138 481-4

[19] Wang C C, Cui Y M and Zhang L W 2007 Appl. Phys. Lett. 90 012904

[20] Schrettle F, Lunkenheimer P, Hemberger J, Ivanov V Y, Mukhin A A, Balbashov A M and Loidl A 2009 Phys. Rev. Lett. 102207208

[21] Ferreira W S et al 2009 Phys. Rev. B 79054303

[22] Samantara S et al 2013 J. Magn. Magn. Mater. 339 168-74

[23] Izquierdo J L, Bolaños G, Zapata V H and Morán O 2014 Curr. Appl. Phys. 14 1492-7

[24] Xu J, Cui Y and Xu H 2014 Ceram. Int. 40 12193-8

[25] Vilarinho R, Almeida A, Machado da Silva J M, Oliveira J B, Sá M A, Tavares P B and Agostinho Moreira J 2015 Solid State Commun. 208 34-40

[26] Xu J, Cui Y and Xu H 2016 Results Phys. 6 811-6

[27] Pal A, Dhana Sekhar C, Venimadhav A, Prellier W and Murugavel P 2018 J. Appl. Phys. 123014102

[28] Pal A, Prellier W and Murugavel P 2018 J. Phys.: Condens. Matter. 30125801

[29] Staruch M, Violette D and Jain M 2013 Mater. Chem. Phys. $139897-900$

[30] Schiemer J, O'Flynn D, Balakrishnan G and Carpenter M A 2013 Phys. Rev. B 88054108

[31] Thomson R I, Chatterji T, Howard C J, Palstra T T M and Carpenter M A 2014 J. Phys.: Condens. Matter. 26045901

[32] Migliori A and Sarrao J L 1997 Resonant Ultrasound Spectroscopy: Applications to Physics, Materials Measurements and Nondestructive Evaluation (New York: Wiley)

[33] Carpenter M A 2015 J. Phys.: Condens. Matter. 27263201

[34] Kasper N V and Troyanchuk I O 1996 J. Phys. Chem. Solids 57 1601-7

[35] Quezel S, Tcheou F, Rossat-Mignod J, Quezel G and Roudaut E 1977 Physica B 86-88 916-8

[36] Kimura T, Goto T, Shintani H, Ishizaka K, Arima T and Tokura Y 2003 Nature 426 55-8

[37] Kajimoto R, Yoshizawa H, Shintani H, Kimura T and Tokura Y 2004 Phys. Rev. B 70012401

[38] Kenzelmann M et al 2005 Phys. Rev. Lett. 95087206

[39] Arima T, Goto T, Yamasaki Y, Miyasaka S, Ishii K, Tsubota M, Inami T, Murakami Y and Tokura Y 2005 Phys. Rev. B 72 100102(R)

[40] Mufti N, Nugrohon A A, Blake G R and Palstra T T M 2008 Phys. Rev. B 78024109

[41] Hemberger J, Lobina S, Krug von Nidda H-A, Tristan N, Ivanov V Y, Mukhin A A, Balbashov A M and Loidl A 2004 Phys. Rev. B 70024414

[42] Wagh A A, Suresh K G, Anil Kumar P S and Elizabeth S 2015 J. Phys. D: Appl. Phys. 48135001

[43] Kuwahara H, Noda K, Nagayama J and Nakamura S 2005 Physica B 359-361 1279-81

[44] Kuwahara H, Akaki M, Tozawa J, Hitomi M, Noda K and Akahoshi D 2009 J. Phys.: Conf. Ser. 150042106

[45] Lin L, Li L, Yan Z B, Tao Y M, Dong S and Liu J-M 2013 Appl. Phys. A 112 947-54

[46] Li L, Lin L, Yan Z B, He Q Y and Liu J-M 2012 J. Appl. Phys. 112034115

[47] Baier J, Meier D, Berggold K, Hemberger J, Balbashov A, Mydosh J A and Lorenz T 2006 Phys. Rev. B 73 100402(R)

[48] Aliouane N et al 2008 J. Phys.: Condens. Matter. 20434215 
[49] Feyerherm R et al 2009 Phys. Rev. B 79134426

[50] Prokhnenko O, Feyerherm R, Mostovoy M, Aliouane N, Dudzik E, Maljuk A and Argyriou D N 2007 Phys. Rev. Lett. 99177206

[51] Handayani I P, Tobey R I, Janusonis J, Mazurenko D A, Mufti N, Nugroho A A, Tjia M O, Palstra T T M and van Loosdrecht P H M 2013 J. Phys.: Condens. Matter. 25 116007

[52] O'Flynn D, Lees M R and Balakrishnan G 2014 J. Phys.: Condens. Matter. 26256002

[53] Pal A, Dhana Sekhar C, Venimadhav A and Murugavel P 2017 J. Phys.: Condens. Matter. 29405803

[54] Mihalik M et al 2017 Physica B 506 163-7

[55] Vilarinho R et al 2017 J. Magn. Magn. Mater. 439 167-72

[56] Vilarinho R, Passos D J, Queirós E C, Tavares P B, Almeida A, Weber M C, Guennou M, Kreisel J and Agostinho Moreira J 2018 Phys. Rev. B 97144110

[57] O'Flynn D, Tomy C V, Lees M R, Daoud-Aladine A and Balakrishnan G 2010 J. Phys.: Conf. Ser. 200012149

[58] O'Flynn D, Tomy C V, Lees M R, Daoud-Aladine A and Balakrishnan G 2011 Phys. Rev. B 83174426

[59] McKnight R E A, Carpenter M A, Darling T W, Buckley A and Taylor P A 2007 Am. Mineral. 92 1665-72

[60] Schiemer J, Spalek L J, Saxena S S, Panagopoulos C, Katsufuji T, Bussmann-Holder A, Köhler J and Carpenter M A 2016 Phys. Rev. B 93054108

[61] Weller M, Li G Y, Zhang J X, Kê T S and Diehl J 1981 Acta Metall. 29 1047-54

[62] Schaller R, Fantozzi G and Grenaud G 2001 Mechanical Spectroscopy $Q^{-1}$ 2001: With Applications to Materials Science (Clausthal: Trans Tech. Publications)

[63] Carpenter M A and Howard C J 2009 Acta Crystallogr. B 65 147-59

[64] Meier D 2006 Diplomarbeit University of Cologne

[65] Meier D, Aliouane N, Argyriou D N, Mydosh J A and Lorenz T 2007 New J. Phys. 9100

[66] Berggold K, Baier J, Meier D, Mydosh J A, Lorenz T, Hemberger J, Balbashov A, Aliouane N and Argyriou D N 2007 Phys. Rev. B 76094418

[67] Mansouri S, Jandl S, Mukhin A, Ivanov V Y and Balbashov A 2017 Sci. Rep. 713796

[68] Chatterji T, Ouladdiaf B and Bhattacharya D 2009 J. Phys.: Condens. Matter. 21306001

[69] Kumar A, Yusuf S M and Ritter C 2017 Phys. Rev. B 96014427

[70] Slonczewski J C and Thomas H 1970 Phys. Rev. B 13599

[71] Thomson R I, Chatterji T and Carpenter M A 2014 J. Phys.: Condens. Matter. 26146001

[72] Carpenter M A and Salje E K H 1998 Eur. J. Mineral. 10 693-812

[73] Cuartero V, Blasco J, Rodríguez-Velamazán J A, García J, Subías G, Ritter C, Stankiewicz J and Canadillas-Delgado L 2012 Phys. Rev. B 86104413

[74] Mahana S, Manju U and Topwal D 2017 J. Phys. D: Appl. Phys. 50035002

[75] Kagawa F, Mochizuki M, Onose Y, Murakawa H, Kaneko Y, Furukawa N and Tokura Y 2009 Phys. Rev. Lett. 102 057604

[76] Farnsworth S M, Kisi E H and Carpenter M A 2011 Phys. Rev. B 84174124

[77] Salje E K H, Carpenter M A, Nataf G F, Picht G, Webber K, Weerasinghe J, Lisenkov S and Bellaiche L 2013 Phys. Rev. B 87014106
[78] Nataf G F, Li Q, Liu Y, Withers R L, Driver S L and Carpenter M A 2013 J. Appl. Phys. 113124102

[79] Aktas O, Salje E K H, Crossley S, Lampronti G I, Whatmore R W, Mathur N D and Carpenter M A 2013 Phys. Rev. B 88 174112

[80] Vilarinho R, Queirós E C, Almeida A, Tavares P B, Guennou M, Kreisel J and Agostinho Moreira J 2015 J. Solid State Chem. 228 76-81

[81] Pal A and Murugavel P 2018 J. Appl. Phys. 123234102

[82] Zhang X et al 2014 Appl. Phys. Lett. 104062903

[83] Perks N J, Zhang Z, Harrison R J and Carpenter M A 2014 J. Phys.: Condens. Matter. 26505402

[84] Zhang Z, Koppensteiner J, Schranz W, Betts J B, Migliori A and Carpenter M A 2010 Phys. Rev. B 82014113

[85] Bidault O, Maglione M, Actis M, Kchikech M and Salce B 1995 Phys. Rev. B 52 4191-7

[86] Aktas O, Crossley S, Carpenter M A and Salje E K H 2014 Phys. Rev. B 90165309

[87] Salce B, Gravil J L and Boatner L A 1994 J. Phys.: Condens. Matter. 6 4077-92

[88] Carpenter M A, McKnight R E A, Howard C J, Zhou Q, Kennedy B J and Knight K S 2009 Phys. Rev. B 80 214101

[89] Venkatesan S, Daumont C, Kooi B J, Noheda B and De Hosso J T M 2009 Phys. Rev. B 80214111

[90] Daumont C J M, Mannix D, Venkatesan S, Catalan G, Rubi D, Kooi B J, De Hosson J T M and Noheda B 2009 J. Phys.: Condens. Matter. 21182001

[91] Déchamps M, de Leon Guevara A M, Pinsard L and Revcolevschi A 2000 Phil. Mag. A 80 119-27

[92] Carpenter M A, Becerro A I and Seifert F 2001 Am. Mineral. $86348-63$

[93] Vasquez J A C, Téllez D A L, Collazos C A and Rojas J R 2016 J. Phys.: Conf. Ser. 687012087

[94] Alonso J A, Martínez-Lope M J, Casais M T and FernándezDíaz M T 2000 Inorg. Chem. 39 917-23

[95] Vasylechko L, Trots D M, Senyshyn A and Lukasiewicz T(HASYLAB) 2006 Ann. Rep. 1 605-6

[96] Salje E K H, Wruck B and Thomas H 1991 Z. Phys. B 82 399-404

[97] Meyer H-W, Carpenter M A, Graeme-Barber A, Sondergeld P and Schranz W 2000 Eur. J. Mineral. 12 1139-50

[98] Meyer H-W, Marion S, Sondergeld P, Carpenter M A, Knight K S, Redfern S A T and Dove M T 2001 Am. Mineral. 86 566-77

[99] Sondergeld P, Schranz W, Kityk A V, Carpenter M A and Libowitzky E 2000 Phase Transit. 71 189-203

[100] Carpenter M A, Meyer H-W, Sondergeld P, Marion S and Knight K S 2003 Am. Mineral. 88 534-46

[101] Agostinho Moreira J et al 2010 Phys. Rev. B 82094418

[102] Carpenter M A 2007 Am. Mineral. 92 309-27

[103] Carpenter M A, Sinogeikin S V and Bass J D 2010 J. Phys.: Condens. Matter. 22035404

[104] Carpenter M A, McKnight R E A, Howard C J and Knight K S 2010 Phys. Rev. B 82094101

[105] Aoyama T, Yamauchi K, Iyama A, Picozzi S, Shimizu K and Kimura T 2014 Nat. Commun. 54927

[106] Muñoz A, Alonso J A, Martínez-Lope M J, García-Muñoz J L and Fernández-Díaz M T 2000 J. Phys.: Condens. Matter. 12 1361-76

[107] Blasco J, Ritter C, García J, de Teresa J M, Pérez-Cacho J and Ibarra M R 2000 Phys. Rev. B 62 5609-18 